\title{
On the motion of slightly rarefied gas induced by a discontinuous surface temperature
}

\author{
$\operatorname{AUTHOR}(\mathrm{S})$ : \\ Taguchi, Satoshi; Tsuji, Tetsuro
}

\section{CITATION:}

Taguchi, Satoshi ... [et al]. On the motion of slightly rarefied gas induced by a discontinuous surface temperature. Journal of Fluid Mechanics 2020, 897: A16.

\section{ISSUE DATE:}

2020-08-25

URL:

http://hdl.handle.net/2433/252787

\section{RIGHT:}

This article has been published in a revised form in Journal of Fluid Mechanics http://doi.org/10.1017/ffm.2020.332. This version is free to view and download for private research and study only. Not for re-distribution or re-use. (๑) The

Author(s), 2020:; The full-text file will be made open to the public on 11 January 2021 in accordance with publisher's 'Terms and Conditions for Self-Archiving'., この論文は出版社版でありません。引用の際には出版社版をご確認ご利用 ください。; This is not the published version. Please cite only the published version. 


\title{
On the motion of slightly rarefied gas induced by a discontinuous surface temperature
}

\author{
Satoshi Taguchi ${ }^{1,2} \dagger$, and Tetsuro Tsuji ${ }^{1,2}$ \\ ${ }^{1}$ Department of Advanced Mathematical Sciences, Graduate School of Informatics, Kyoto \\ University, Kyoto 606-8501, Japan \\ ${ }^{2}$ Research Project of Fluid Science and Engineering, Advanced Engineering Research Center, \\ Kyoto University
}

(Received xx; revised xx; accepted xx)

The motion of a slightly rarefied gas in a long and straight two-dimensional channel caused by a discontinuous surface temperature is investigated on the basis of kinetic theory with a special interest in the fluid-dynamic description. More precisely, the channel is longitudinally divided into two parts and each part is kept at a uniform temperature different each other, so that the surface temperature of the whole channel has a jump discontinuity at the junction. Under the assumption that the amount of jump in the surface temperature is small, the steady behavior of the gas induced in the channel is studied on the basis of the linearized Boltzmann equation and the diffuse reflection boundary condition in the case where the Knudsen number, defined by the ratio of the molecular mean free path and the width of the channel, is small. Using a matched asymptotic expansion method combined with Sone's asymptotics, a Stokes system describing the overall macroscopic behavior of the gas inside the channel is derived, with a new feature of the "slip boundary condition" for the flow velocity due to the jump discontinuity in the surface temperature of the channel. This condition takes the form of a diverging singularity with source and sink located at the point of discontinuity, with a multiplicative factor determined through the analysis of a spatially two-dimensional Knudsen-layer (or a Knudsen-zone) problem. Some numerical demonstrations based on the Bhatnagar-Gross-Krook (BGK) equation are also presented.

Key words:

\section{Introduction}

In a gas in small scale systems or in a gas with low pressure, the molecular mean free path is often no longer negligible in comparison with the characteristic system size. In such a gas (or in a rarefied gas), the temperature field causes a steady motion of the gas in the absence of external forces (e.g., gravity). A well-known example is the thermal creep flow (or thermal transpiration) induced over a surface with a nonuniform temperature distribution (Kennard 1938; Sone 1966; Sone \& Yamamoto 1968; Loyalka 1969; Niimi 1971; Ohwada et al. 1989a,b; Sharipov 2002; Takata \& Funagane 2011). The thermally

$\dagger$ Email address for correspondence: taguchi.satoshi.5a@kyoto-u.ac.jp 
induced flows have been an active research area of rarefied gas dynamics (or kinetic theory of gases) in the last half-century (see Sone (2007) and the references therein).

We shall hereafter focus on steady flows. As a kinetic effect, one requires kinetic theory (i.e., the Boltzmann equation) for accurate descriptions of the thermally induced flows. However, when the molecular mean free path is sufficiently small compared with the characteristic size of the system (i.e., near-continuum regime), a macroscopic system can be derived from the Boltzmann system and the thermally induced flows are conveniently described by suitable fluid-dynamic-type equations and their boundary conditions. This theory, not only intended to cover the thermally induced flows but also to cover other kinetic effects such as the shear slip, the temperature jump, and so on, is called the generalized slip flow (GSF) theory, which was developed notably by Sone (Sone 1969, 1971, 2002, 2007). The theory has been applied to various thermally induced flows (e.g., Sone 2007; Li et al. 2014).

The GSF theory assumes that the boundary shape of bodies and the boundary condition on the bodies are smooth. To be more specific, the latter means that the surface temperature as well as the surface velocity of each of the body should be a smooth function of the position on the body. Therefore, the theory does not apply to the case where the boundary temperature has a jump discontinuity and/or to the case where the boundary shape has a sharp edge.

In the meantime, thermally induced flows caused by a discontinuous surface temperature and/or a sharp edge have also been investigated in the literature. For example, the thermal edge flow, induced around a uniformly heated flat plate with a sharp edge, was discovered (Aoki et al. 1995; Sone \& Yoshimoto 1997), and applied to a vacuum pump (the thermal edge pump; Sugimoto \& Sone (2005); Sone (2007)). Also, flows induced over a flat plate with different surface temperatures on both sides were investigated (Taguchi \& Aoki 2012; Ketsdever et al. 2012) in connection with thermophoretic effects (Ketsdever et al. 2012). It was shown that the parallel alignment of flat plates also have a pumping effect when appropriately heated (Taguchi \& Aoki 2015; Baier et al. 2017). Actually, flows related to discontinuous surface temperature have been a subject of recent studies such as the thermally-driven pumps (e.g., Donkov et al. 2011; Lotfian \& Roohi 2019) and the thermophoresis (Aoki et al. 2014) (see also Baier et al. (2018)). Despite its theoretical and practical interest, however, the understanding of these flows is far behind due to the lack of general theory.

Given these situations, it is important to examine whether one can extend the GSF theory to the case of a discontinuous surface temperature and/or a non-smooth boundary shape. The purpose of the present paper is to give an affirmative answer to this question in the simplest case. More specifically, we consider a slightly rarefied gas in a long and straight two-dimensional channel whose surface temperature has a jump discontinuity. With the assumption of small jumps, we derive a system of Stokes equation for the overall (stationary) flow field in the channel together with a slip boundary condition, which is intended to describe a flow due to the discontinuous surface temperature. This slip condition, however, is far from the conventional slip condition and takes the form of a source/sink singularity which diverges in approaching the point of discontinuity.

The rest of the paper is organized as follows. In $\S 2$, as a preparation for the subsequent analysis, we consider a rarefied gas flow between two parallel plates with a smooth surface temperature distribution, and investigate its asymptotic behavior for small Knudsen numbers using conventional Sone's method. In $\S 3$, we consider a rarefied gas flow between two parallel plates with a discontinuous surface temperature and discuss its asymptotic behavior for small Knudsen numbers based on the result of $\S 2$. We show that Sone's asymptotics obtained in $\S 2$ breaks down in the vicinity of the discontinuous 
On the motion of slightly rarefied gas induced by a discontinuous surface temperature3

point, and therefore a local correction is needed there. We use this local correction to derive the desired boundary condition for the flow velocity. $\S 4$ presents some numerical demonstrations to support our analytical prediction. $\S 5$ is the concluding remarks.

\section{Behavior of a slightly rarefied gas between two parallel plates with a smooth temperature distribution}

As preliminary, we consider a gas confined between two parallel plates with a smooth temperature distribution and investigate its behavior for small Knudsen numbers. In the followings, we denote the characteristic length by $L$, the characteristic density of the gas by $\rho_{0}$, the characteristic temperature by $T_{0}$, and the characteristic pressure by $p_{0}=\rho_{0} R T_{0}$, where $R$ is the specific gas constant, i.e., $R=k_{B} / m$ with $k_{B}$ and $m$ being the Boltzmann constant and the mass of a molecule, respectively.

\subsection{Problem}

Let $L x_{i}(i=1,2,3)$ be the space rectangular coordinate system. We consider a rarefied gas confined between two parallel plates located at $L x_{1}= \pm L a$, where $a$ is a positive constant. The temperatures of the two plates, which are constant in time, are the same and the temperature distribution is given by $T_{0}\left(1+\tau_{\mathrm{w}}\right)$, where $\tau_{\mathrm{w}}=\tau_{\mathrm{w}}\left(x_{2}, x_{3}\right)$ is assumed to be a smooth function of $x_{2}$ and $x_{3}$. There is no pressure gradient imposed on the gas nor external force. Investigate the steady behavior of the gas in the domain $D=$ $\left\{\left(x_{1}, x_{2}, x_{3}\right) \mid-a<x_{1}<a,-\infty<x_{2}<\infty,-\infty<x_{3}<\infty\right\}$ based on the Boltzmann equation with the diffuse reflection boundary condition on the plate surface, under the assumption that $\left|\partial \tau_{\mathrm{w}} / \partial x_{i}\right|$ is so small that the equation and boundary conditions can be linearized around the reference equilibrium state at rest. In particular, we investigate the steady behavior of the gas when the Knudsen number of the system $K n=\ell_{0} / L$ is small. Here, $\ell_{0}$ is the mean free path of the gas molecules in the equilibrium state at rest with temperature $T_{0}$ and density $\rho_{0}$. Throughout the paper, we use the symbol

$$
\varepsilon=\frac{\sqrt{\pi}}{2} K n
$$

to denote the small parameter of the problem.

To illustrate the physical situation that can be described by the above problem, we give two particular examples for $\tau_{\mathrm{w}}$. The simplest example is the case where $\partial \tau_{\mathrm{w}} / \partial x_{i}$ is constant, say $\left(\partial \tau_{\mathrm{w}} / \partial x_{2}, \partial \tau_{\mathrm{w}} / \partial x_{3}\right)=\left(\beta_{1}, 0\right)$ with $\beta_{1}$ being a (small) constant. In this case, the problem is nothing but the classical thermal transpiration between two parallel plates (Ohwada et al. 1989a; Niimi 1971; Sone 2007). Another example is given by a periodic function, say $\tau_{\mathrm{w}}=\beta_{2} g\left(x_{2}\right)$, where $\beta_{2}$ is a (small) constant and $g\left(x_{2}\right)$ with $d g / d x_{2}=O(1)$ is periodic in $x_{2}$. In this case, we are concerned with the spatially periodic motion of a gas thermally induced in the channel.

\subsection{Basic equations}

Let the molecular velocity be denoted by $\left(2 R T_{0}\right)^{1 / 2} \zeta_{i}$ and the velocity distribution function by $\rho_{0}\left(2 R T_{0}\right)^{-3 / 2}\left(1+\phi\left(x_{i}, \zeta_{i}\right)\right) E$, where $E=E\left(\zeta_{i}\right):=\pi^{-3 / 2} \exp \left(-\zeta_{j}^{2}\right)$ is the normalized absolute Maxwellian. The linearized Boltzmann equation for the present steady problem is then written as

$$
\zeta_{i} \partial_{i} \phi=\frac{1}{\varepsilon} \mathcal{L}(\phi)
$$


where $\partial_{i}=\partial / \partial x_{i}$ and $\mathcal{L}$ is the linearized collision operator, whose explicit form is omitted here (see, e.g., Sone 2007, Chap. 1). The diffuse reflection boundary condition on the plates are given as follows:

$$
\begin{aligned}
\phi=2 \sqrt{\pi} \int_{\zeta_{1} \lessgtr 0}\left|\zeta_{1}\right| \phi E \mathrm{~d} \boldsymbol{\zeta}+\left(\zeta_{j}^{2}-2\right) \tau_{\mathrm{w}}, \quad \zeta_{1} \gtrless 0, \\
\left(x_{1}=\mp a,-\infty<x_{2}<\infty,-\infty<x_{3}<\infty\right),
\end{aligned}
$$

where $\mathrm{d} \boldsymbol{\zeta}=\mathrm{d} \zeta_{1} \mathrm{~d} \zeta_{2} \mathrm{~d} \zeta_{3}$.

Next, we introduce the macroscopic quantities. Let $\rho_{0}\left(1+\omega\left(x_{i}\right)\right)$ denote the mass density, $\left(2 R T_{0}\right)^{1 / 2} u_{i}\left(x_{i}\right)$ the flow velocity, $T_{0}\left(1+\tau\left(x_{i}\right)\right)$ the temperature, and $p_{0}\left(1+P\left(x_{i}\right)\right)$ the pressure of the gas. Then, $\omega, u_{i}, \tau$, and $P$ are expressed in terms of $\phi$ as the moments with respect to the molecular velocity, i.e.,

$$
\begin{aligned}
\omega & =\langle\phi\rangle, \quad u_{i}=\left\langle\zeta_{i} \phi\right\rangle, \\
\tau & =\frac{2}{3}\left\langle\left(\zeta_{j}^{2}-\frac{3}{2}\right) \phi\right\rangle, \quad P=\frac{2}{3}\left\langle\zeta_{j}^{2} \phi\right\rangle=\omega+\tau .
\end{aligned}
$$

Here, the bracket symbol means the following integral:

$$
\langle f\rangle=\int f\left(\zeta_{i}\right) E \mathrm{~d} \boldsymbol{\zeta}
$$

where the range of integration spans over the whole velocity space.

\subsection{Summary of the asymptotic analysis for small $\varepsilon$}

We investigate the behavior of the gas between the plates in the case where $\varepsilon \ll 1$, following the method of Sone (the asymptotic analysis of the Boltzmann equation for small $\varepsilon$ (Sone 2002, 2007)). Since the procedure is explained in detail in the references, we briefly explain the derivation of the fluid-dynamic-type system to the first order of $\varepsilon$ for the subsequent discussions.

\subsubsection{Hilbert solution and fluid-dynamic-type equations}

First, putting aside the boundary condition, we consider the solution of the linearized Boltzmann equation (2.2) which varies moderately in space. This type of solution is called the Hilbert solution and is designated by attaching the subscript $H$, i.e., $\partial_{i} \phi_{H}=O\left(\phi_{H}\right)$. We seek $\phi_{H}$ in the form of a simple expansion in $\varepsilon$ :

$$
\phi_{H}=\phi_{H 0}+\phi_{H 1} \varepsilon+\cdots .
$$

Corresponding to this expansion, the macroscopic quantities are also expanded in a power series of $\varepsilon$ :

$$
h_{H}=h_{H 0}+h_{H 1} \varepsilon+\cdots, \quad\left(h=\omega, u_{i}, \tau, P\right) .
$$

The relation between $h_{H m}$ and $\phi_{H m}(m=0,1, \ldots)$ is simply obtained as follows:

$$
\begin{aligned}
\omega_{H m} & =\left\langle\phi_{H m}\right\rangle, \quad u_{i H m}=\left\langle\zeta_{i} \phi_{H m}\right\rangle, \\
\tau_{H m} & =\frac{2}{3}\left\langle\left(\zeta_{j}^{2}-\frac{3}{2}\right) \phi_{H m}\right\rangle, \quad P_{H m}=\frac{2}{3}\left\langle\zeta_{j}^{2} \phi_{H m}\right\rangle .
\end{aligned}
$$

Substituting the above expansion for $\phi_{H}$ into (2.2) and arranging the terms of the same order in $\varepsilon$, we obtain the following sequence of linear integral equations for $\phi_{H m}$ :

$$
\begin{aligned}
& \mathcal{L}\left(\phi_{H 0}\right)=0, \\
& \mathcal{L}\left(\phi_{H m}\right)=\zeta_{i} \partial_{i} \phi_{H m-1}, \quad(m \geqslant 1) .
\end{aligned}
$$


On the motion of slightly rarefied gas induced by a discontinuous surface temperature5

These equations can be solved successively from the lowest order, provided that the following solvability conditions are satisfied (Sone 2002, 2007):

$$
\partial_{i}\left\langle\psi_{j} \zeta_{i} \phi_{H m-1}\right\rangle=0, \quad(m=1,2, \ldots) .
$$

Here, $j \in\{0,1, \cdots, 4\}$ and $\left(\psi_{0}, \psi_{i}, \psi_{4}\right)=\left(1, \zeta_{i}, \zeta_{j}^{2}\right)$ are the collision invariants. These solvability conditions provide closed sets of partial differential equations for the macroscopic variables (i.e., fluid-dynamic-type equations). Specifically, the resulting equations are the well-known Stokes set of equations (Sone 2002, 2007), which are summarized as follows:

$$
\begin{aligned}
& \partial_{i} P_{H 0}=0, \\
& \partial_{i} u_{i H m}=0, \\
& \gamma_{1} \nabla^{2} u_{i H m}-\partial_{i} P_{H m+1}=0, \\
& \nabla^{2} \tau_{H m}=0, \\
& (m=1,2, \ldots) .
\end{aligned}
$$

Here, $\gamma_{1}$ is the constant (dimensionless viscosity) defined by $\gamma_{1}=(2 / 15)\left\langle\zeta_{j}^{2} \zeta_{k}^{2} B\right\rangle$, where $B=B(\zeta)$ is the function introduced below in $(2.17 b)$. The numerical value of $\gamma_{1}$ depends on the molecular model. For example, for the hard-sphere model (HS), the Bhatnagar-Gross-Krook (BGK) model (Bhatnagar et al. 1954; Welander 1954), and for the ellipsoidal statistical (ES) model (Holway 1966; Andries et al. 2000; Brull 2015), the values are given by

$$
\gamma_{1}= \begin{cases}1.270042427, & (\mathrm{HS}), \\ 1, & (\mathrm{BGK}), \\ \operatorname{Pr}, & (\mathrm{ES}),\end{cases}
$$

where $\operatorname{Pr}$ is a model parameter whose physical meaning is the Prandtl number. The ES model reduces to the BGK model when $\operatorname{Pr}=1$.

It is worth noting that the density of the gas is determined by the equation of state

$$
\omega_{H m}=P_{H m}-\tau_{H m}, \quad(m=0,1,2, \ldots) .
$$

Therefore, the leading-order density $\omega_{H 0}$ is not uniform if the leading-order temperature $\tau_{H 0}$ is not uniform. In this sense, the fluid is not really "incompressible" although the flow velocity is determined by the Stokes equation for an incompressible fluid.

The solvability conditions being satisfied, the velocity distribution function $\phi_{H m}$ 's are expressed in terms of $\left(P_{H m}, u_{i H m}, \tau_{H m}\right)$ and the spatial derivatives of $\left(P_{H n}, u_{i H n}, \tau_{H n}\right)$ $(n<m)$. For instance, $\phi_{H 0}$ and $\phi_{H 1}$ are given as follows:

$$
\begin{aligned}
& \phi_{H 0}=\phi_{e H 0}, \\
& \phi_{H 1}=\phi_{e H 1}-\zeta_{i} A(\zeta)\left(\partial_{i} \tau_{H 0}\right)-\frac{1}{2} \zeta_{i} \zeta_{j} B(\zeta)\left(\partial_{j} u_{i H 0}+\partial_{i} u_{j H 0}\right),
\end{aligned}
$$

where $\zeta=\left(\zeta_{j}^{2}\right)^{1 / 2}$,

$$
\phi_{e H m}=P_{H m}+2 \zeta_{i} u_{i H m}+\left(\zeta_{j}^{2}-\frac{5}{2}\right) \tau_{H m},
$$


and $A=A(\zeta)$ and $B=B(\zeta)$ are the solutions to the following integral equations:

$$
\begin{aligned}
& \mathcal{L}\left(\zeta_{i} A\right)=-\zeta_{i}\left(\zeta^{2}-\frac{5}{2}\right), \quad \text { with }\left\langle\zeta^{2} A\right\rangle=0, \\
& \mathcal{L}\left(\left(\zeta_{i} \zeta_{j}-\frac{\zeta^{2}}{3} \delta_{i j}\right) B\right)=-2\left(\zeta_{i} \zeta_{j}-\frac{\zeta^{2}}{3} \delta_{i j}\right),
\end{aligned}
$$

where $\delta_{i j}$ is the Kronecker delta.

\subsubsection{The Knudsen-layer analysis and the boundary conditions for the fluid-dynamic-type equations}

In the discussion of the Hilbert solution, the boundary condition has not been taken into account. Let us suppose that the leading-order flow velocity $u_{i H 0}$ and temperature $\tau_{H 0}$ take the following values on the boundary:

$$
u_{i H 0}=0, \quad \tau_{H 0}=\tau_{\mathrm{w}}, \quad\left(x_{1}=\mp a,-\infty<x_{2}<\infty,-\infty<x_{3}<\infty\right) .
$$

Then, $\phi_{H 0}$ being the local Maxwellian, it satisfies the diffuse reflection boundary condition $(2.3)$ on $x_{1}=\mp a$. However, for higher orders, $\phi_{H m}(m \geqslant 1)$ cannot be made to satisfy the boundary condition. This is because the Hilbert solution was obtained under the restriction of moderate variations in all spatial directions. Therefore, to construct the solution satisfying the boundary condition, we need to introduce a boundary layer in which the solution is allowed to change abruptly in the direction normal to the boundary. This boundary layer adjacent to the walls $x_{1}=\mp a$ is called the Knudsen layer and the correction to the Hilbert solution in the Knudsen layer is called the Knudsen-layer correction (Sone 2002, 2007).

As is well known, the so-called slip/jump boundary conditions are derived from the Knudsen-layer analysis, whose general procedure is explained in detail in Sone (2007, 2002). In the present study, we repeat the derivation because we will require the information on the velocity distribution function in the Knudsen layer.

We seek the solution in the form

$$
\phi=\phi_{H}+\phi_{K}
$$

Here, $\phi_{K}$ represents the correction to the Hilbert solution (Knudsen-layer correction) and the length scale of variation of $\phi_{K}$ in the direction normal to the boundary is assumed to be of the order of $\varepsilon$, i.e.,

$$
\partial_{1} \phi_{K}=O\left(\frac{\phi_{K}}{\varepsilon}\right)
$$

In order to analyze the Knudsen layers adjacent to the boundary $x_{1}=-a$ and $x_{1}=a$ in a unified way, we introduce new variables $\left(\eta, \zeta_{n}\right)$ by

$$
x_{1}=\mp a \pm \varepsilon \eta, \quad \zeta_{n}= \pm \zeta_{1}, \quad\left(\text { near } x_{1}=\mp a\right),
$$

where $\eta(\geqslant 0)$ is the stretched coordinate in the direction normal to the boundary. With these variables, we put

$$
\phi_{K}=\phi_{K}\left(\eta, x_{2}, x_{3}, \zeta_{n}, \zeta_{2}, \zeta_{3}\right)
$$

Then, $\phi_{K}$ satisfies the following equation:

$$
\zeta_{n} \partial_{\eta} \phi_{K}-\mathcal{L}\left(\phi_{K}\right)=-\varepsilon\left(\zeta_{2} \partial_{2}+\zeta_{3} \partial_{3}\right) \phi_{K},
$$

where $\partial_{\eta}=\partial / \partial \eta$. We further expand $\phi_{K}$ in a power series of $\varepsilon$, i.e.,

$$
\phi_{K}=\phi_{K 1} \varepsilon+\phi_{K 2} \varepsilon^{2}+\cdots \text {. }
$$


On the motion of slightly rarefied gas induced by a discontinuous surface temperature 7

Note that the expansion starts from $\varepsilon$ order, since there is no correction required for $\phi_{H 0}$ and thus $\phi_{K}=O(\varepsilon)$. Substituting this expansion into equation (2.23) and arranging the terms of the same order of $\varepsilon$, one can derive a sequence of equations for $\phi_{K m}$. On the other hand, the boundary condition for $\phi_{K m}$ at $\eta=0$ is obtained from the requirement that $\phi_{H m}+\phi_{K m}$ satisfies the diffuse reflection boundary condition (2.3). Finally, we require that $\phi_{K m}$ approaches zero rapidly as $\eta \rightarrow \infty$ since it is a correction (matching condition).

We summarize the equation and boundary condition for $\phi_{K 1}$ thus obtained:

$$
\begin{aligned}
\zeta_{n} \partial_{\eta} \phi_{K 1} & =\mathcal{L}\left(\phi_{K 1}\right), \\
\phi_{K 1} & =-\left(\zeta^{2}-2\right)\left(\tau_{H 1}\right)_{0}+\zeta_{n} A(\zeta) n_{i}\left(\partial_{i} \tau_{H 0}\right)_{0} \\
& +\bar{\zeta}_{i}\left[-2\left(u_{i H 1}\right)_{0}+A(\zeta)\left(\partial_{i} \tau_{H 0}\right)_{0}+\zeta_{n} B(\zeta) n_{j}\left(\partial_{j} u_{i H 0}+\partial_{i} u_{j H 0}\right)_{0}\right] \\
& +K\left(\phi_{K 1}\right), \quad \zeta_{n}>0, \quad \eta=0, \\
\phi_{K 1} & \rightarrow 0, \quad \text { (rapidly), } \quad \eta \rightarrow \infty .
\end{aligned}
$$

Here, $n_{i}=( \pm 1,0,0)$ is the unit normal vector on the boundary $x_{1}=\mp a$ pointing to the gas, $\bar{\zeta}_{i}=\zeta_{i}-\zeta_{n} n_{i}=\zeta_{j}\left(\delta_{i j}-n_{i} n_{j}\right)$, and the symbol $(\cdot)_{0}$ indicates the value on the boundary $\left(\eta=0\right.$ or $\left.x_{1}=\mp a\right)$. Further, we have introduced the (linear) operator

$$
K(\varphi)=2 \sqrt{\pi} \int_{\zeta_{1}<0}\left|\zeta_{1}\right| \varphi\left(\zeta_{1}, \zeta_{2}, \zeta_{3}\right) E \mathrm{~d} \boldsymbol{\zeta},
$$

for later convenience. Incidentally, the impermeability on the boundary implies

$$
\left(u_{1 H 1}\right)_{0}=0 .
$$

The boundary condition (2.26) was simplified with the aid of this condition.

The linearity of the problem and the axysimmetry of the operators $\zeta_{n} \partial_{\eta}-\mathcal{L}$ and $K$ with respect to the $\eta$ axis allow us to seek $\phi_{K 1}$ in the form:

$$
\begin{aligned}
\phi_{K 1} & =\varphi_{1}^{(0)}\left(\eta, \zeta_{n}, \zeta\right) n_{i}\left(\partial_{i} \tau_{H 0}\right)_{0} \\
& +\bar{\zeta}_{i}\left[\varphi_{1}^{(1)}\left(\eta, \zeta_{n}, \zeta\right) n_{j}\left(\partial_{j} u_{i H 0}+\partial_{i} u_{j H 0}\right)_{0}+\varphi_{2}^{(1)}\left(\eta, \zeta_{n}, \zeta\right)\left(\partial_{i} \tau_{H 0}\right)_{0}\right] .
\end{aligned}
$$

Splitting further the constants $\left(\tau_{H 1}\right)_{0}$ and $\left(\delta_{i j}-n_{i} n_{j}\right)\left(u_{j H 1}\right)_{0}$ in $(2.26)$ as

$$
\begin{aligned}
\left(\tau_{H 1}\right)_{0} & =c_{1}^{(0)} n_{i}\left(\partial_{i} \tau_{H 0}\right)_{0} \\
\left(\delta_{i j}-n_{i} n_{j}\right)\left(u_{j H 1}\right)_{0} & =b_{1}^{(1)}\left(\delta_{i j}-n_{i} n_{j}\right) n_{k}\left(\partial_{k} u_{j H 0}+\partial_{j} u_{k H 0}\right)_{0} \\
& +b_{2}^{(2)}\left(\delta_{i j}-n_{i} n_{j}\right)\left(\partial_{j} \tau_{H 0}\right)_{0}
\end{aligned}
$$

we see that $\varphi_{1}^{(0)}=\varphi_{1}^{(0)}\left(\eta, \zeta_{n}, \zeta\right)$ and $\varphi_{j}^{(1)}=\varphi_{j}^{(1)}\left(\eta, \zeta_{n}, \zeta\right)$ solve the following half-space boundary-value problems:

$$
\begin{aligned}
& \left\{\begin{array}{l}
\left(\zeta_{n} \partial_{\eta}-\mathcal{L}\right) \varphi_{1}^{(0)}=0 \\
\varphi_{1}^{(0)}=-\left(\zeta^{2}-2\right) c_{1}^{(0)}+\zeta_{n} A(\zeta)+K\left(\varphi_{1}^{(0)}\right), \quad \zeta_{n}>0, \quad \eta=0, \\
\varphi_{1}^{(0)} \rightarrow 0, \quad \eta \rightarrow \infty
\end{array}\right. \\
& \left\{\begin{array}{l}
\left(\zeta_{n} \partial_{\eta}-\mathcal{L}_{1}\right) \varphi_{j}^{(1)}=-I_{j}\left(\eta, \zeta_{n}, \zeta\right), \\
\varphi_{j}^{(1)}=-2 b_{j}^{(1)}+J_{j}\left(\zeta_{n}, \zeta\right), \quad \zeta_{n}>0, \quad \eta=0, \\
\varphi_{j}^{(1)} \rightarrow 0, \quad \eta \rightarrow \infty
\end{array}\right.
\end{aligned}
$$




$$
\begin{array}{ccccc} 
& \text { Sone }(2002,2007) & \text { HS } & \text { BGK } & \text { Notes } \\
c_{1}^{(0)} & d_{1} & 2.4001 & 1.30272 & \text { Temperature jump } \\
b_{1}^{(1)} & -k_{0} & 1.2540 & 1.01619 & \text { Shear slip } \\
b_{2}^{(1)} & -K_{1} & 0.6465 & 0.38316 & \text { Thermal slip } \\
b_{3}^{(1)} & d_{1} K_{1}-a_{4} & -1.5846 & -0.77837 & \\
c_{1}^{(0)} b_{2}^{(1)}+b_{3}^{(1)} & -a_{4} & -0.0330 & -0.27922 & \text { Thermal stress slip }
\end{array}
$$

TABLE 1. The numerical values of slip/jump coefficients under the diffuse reflection boundary condition for a hard-sphere gas (HS) and for the BGK model (BGK) (Sone 2002, 2007; Takata \& Hattori 2012). The correspondence to the notations in Sone $(2002,2007)$ are also shown.

where $c_{1}^{(0)}$ and $b_{j}^{(1)}$ 's are constants,

$$
\begin{array}{lll}
I_{1}=0, & I_{2}=0, & I_{3}=\varphi_{1}^{(0)}\left(\eta, \zeta_{n}, \zeta\right), \\
J_{1}=\zeta_{n} B(\zeta), & J_{2}=A(\zeta), & J_{3}=2 \zeta_{n} F(\zeta),
\end{array}
$$

and the operator $\mathcal{L}_{1}$ is defined by $\mathcal{L}\left(\bar{\zeta}_{i} g\left(\zeta_{n}, \zeta\right)\right)=\bar{\zeta}_{i} \mathcal{L}_{1}(g)\left(\zeta_{n}, \zeta\right)$. Note that we have also introduced $\varphi_{3}^{(1)}$ for later use and that $F=F(\zeta)$ is the solution to

$$
\mathcal{L}\left(\left(\zeta_{i} \zeta_{j}-\frac{\zeta^{2}}{3} \delta_{i j}\right) F(\zeta)\right)=\left(\zeta_{i} \zeta_{j}-\frac{\zeta^{2}}{3} \delta_{i j}\right) A(\zeta)
$$

These problems form the so-called Knudsen-layer problem and it is known that (i) there exists a solution to the problem if and only if the constant $c_{1}^{(0)}$ or $b_{j}^{(1)}$ takes a special value; (ii) the solution is unique; (iii) the decay of the solution as $\eta \rightarrow \infty$ is exponentially fast (Sone 2007; Bardos et al. 1986; Coron et al. 1988). With the constants $b_{j}^{(1)}(j=1,2)$ and $c_{1}^{(0)}$ thus determined, $\left(\tau_{H 1}\right)_{0}$ and $\left(u_{i H 1}\right)_{0}$ satisfy the following conditions on the boundary:

$$
\begin{aligned}
& \left(\tau_{H 1}\right)_{0}=c_{1}^{(0)} n_{i}\left(\partial_{i} \tau_{H 0}\right)_{0} \\
& \left(u_{i H 1}\right)_{0} n_{i}=0, \quad\left(u_{i H 1}\right)_{0} t_{i}=b_{1}^{(1)} t_{i} n_{j}\left(\partial_{j} u_{i H 0}+\partial_{i} u_{j H 0}\right)_{0}+b_{2}^{(1)} t_{i}\left(\partial_{i} \tau_{H 0}\right)_{0},
\end{aligned}
$$

where $t_{i}$ is an arbitrary unit vector tangent to the boundary. Equations (2.35) and (2.36) provide the consistent boundary conditions for the Stokes set of equations (2.12) for $m=1$.

The constants $b_{1}^{(1)}$ and $b_{2}^{(1)}$ are known as the shear-slip and thermal-slip (or the thermal creep) coefficients, respectively, whereas $c_{1}^{(0)}$ is known as the temperature-jump coefficient. The numerical values of these constants for the hard-sphere model (HS) and the BGK model have been known for a long time (Sone 2002, 2007) and are given in Table 1. For the ES model, the following relations are known to hold (Takata et al. 2016), i.e., $\left(b_{1}^{(1)}\right)_{\mathrm{ES}} / \operatorname{Pr}=\left(b_{1}^{(1)}\right)_{\mathrm{BGK}}$ and $\left(b_{2}^{(1)}\right)_{\mathrm{ES}}=\left(b_{2}^{(1)}\right)_{\mathrm{BGK}}$, where $(\cdot)_{\mathrm{ES}}$ and $(\cdot)_{\mathrm{BGK}}$ mean the values for the ES and BGK models, respectively. The numerical values of $c_{1}^{(0)}$ and $b_{3}^{(1)}$ for the ES model with $\operatorname{Pr}=2 / 3$ can be found in Takata et al. (2016) (see also Takata \& Hattori 2015).

Finally, we present the Knudsen-layer correction for the macroscopic variables. Corresponding to (2.19), the macroscopic quantities $h\left(h=\omega, u_{i}, \tau, P\right)$ are expressed as $h=h_{H}+h_{K}$, where $h_{K}$ is given by (2.4) with $h=h_{K}$ and $\phi=\phi_{K}$. Corresponding to 
On the motion of slightly rarefied gas induced by a discontinuous surface temperature9 the expansion of $\phi_{K}$ in $\varepsilon$, we have

$$
h_{K}=h_{K 1} \varepsilon+h_{K 2} \varepsilon^{2}+\cdots,
$$

with

$$
\begin{aligned}
\omega_{K m} & =\left\langle\phi_{K m}\right\rangle, \quad u_{i K m}=\left\langle\zeta_{i} \phi_{K m}\right\rangle, \\
\tau_{K m} & =\frac{2}{3}\left\langle\left(\zeta_{j}^{2}-\frac{3}{2}\right) \phi_{K m}\right\rangle, \quad P_{K m}=\frac{2}{3}\left\langle\zeta_{j}^{2} \phi_{K m}\right\rangle,
\end{aligned}
$$

$(m=1,2, \ldots)$. Since $\phi_{K 1}$ is expressed by $(2.30)$, the direct substitution into (2.38) yields the following expressions for the macroscopic quantities in the Knudsen layer:

$$
\begin{aligned}
& \omega_{K 1}=\Omega_{1}^{(0)} n_{i}\left(\partial_{i} \tau_{H 0}\right)_{0}, \quad \tau_{K 1}=\Theta_{1}^{(0)} n_{i}\left(\partial_{i} \tau_{H 0}\right)_{0}, \\
& P_{K 1}=\left(\Omega_{1}^{(0)}+\Theta_{1}^{(0)}\right) n_{i}\left(\partial_{i} \tau_{H 0}\right)_{0}=\omega_{K 1}+\tau_{K 1}, \\
& u_{i K 1} n_{i}=0, \quad u_{i K 1} t_{i}=Y_{1}^{(1)} t_{i} n_{j}\left(\partial_{j} u_{i H 0}+\partial_{i} u_{j H 0}\right)_{0}+Y_{2}^{(1)} t_{i}\left(\partial_{i} \tau_{H 0}\right)_{0},
\end{aligned}
$$

where $\Omega_{1}^{(0)}=\Omega_{1}^{(0)}(\eta), \Theta_{1}^{(0)}=\Theta_{1}^{(0)}(\eta)$, and $Y_{j}^{(1)}=Y_{j}^{(1)}(\eta)$ are defined by

$$
\Omega_{1}^{(0)}=\left\langle\varphi_{1}^{(0)}\right\rangle, \quad \Theta_{1}^{(0)}=\frac{2}{3}\left\langle\left(\zeta^{2}-\frac{3}{2}\right) \varphi_{1}^{(0)}\right\rangle, \quad Y_{j}^{(1)}=\frac{1}{2}\left\langle\left(\zeta^{2}-\zeta_{n}^{2}\right) \varphi_{j}^{(1)}\right\rangle .
$$

Note that $\left\langle\zeta_{n} \varphi_{1}^{(0)}\right\rangle=0$ was used in the derivation of $(2.39)$.

\subsection{Summary of Sone's asymptotics}

In this section, we have considered the steady behavior of a rarefied gas between two parallel plates whose temperature distribution is a smooth function of $x_{2}$ and $x_{3}$ in the case where the Knudsen number is small. Its velocity distribution functions are expressed as

$$
\phi=\phi_{H 0}+\varepsilon\left(\phi_{H 1}+\phi_{K 1}\right)+\cdots,
$$

with

$$
\begin{gathered}
\phi_{H 0}=\phi_{e H 0}, \\
\phi_{H 1}=\phi_{e H 1}-\zeta_{i} A(\zeta) \partial_{i} \tau_{H 0}-\frac{1}{2} \zeta_{i} \zeta_{j} B(\zeta)\left(\partial_{i} u_{j H 0}+\partial_{j} u_{i H 0}\right), \\
\phi_{K 1}=\varphi_{1}^{(0)}\left(\eta, \zeta_{n}, \zeta\right) n_{i}\left(\partial_{i} \tau_{H 0}\right)_{0} \\
+\bar{\zeta}_{i} \varphi_{1}^{(1)}\left(\eta, \zeta_{n}, \zeta\right) n_{j}\left(\partial_{j} u_{i H 0}+\partial_{i} u_{j H 0}\right)_{0}+\bar{\zeta}_{i} \varphi_{2}^{(1)}\left(\eta, \zeta_{n}, \zeta\right)\left(\partial_{i} \tau_{H 0}\right)_{0},
\end{gathered}
$$

where $\phi_{e H m}$ is given by (2.16). In these expressions, $A, B, \varphi_{1}^{(0)}$, and $\varphi_{j}^{(1)}$ are universal in the sense that they do not depend on specific problems. On the other hand, the macroscopic variables arising in the velocity distribution functions contain the specific information of the problem under consideration. They are obtained by solving the Stokes system (2.11)-(2.12) under the following boundary conditions on $x_{1}=\mp a$ :

- Order $\varepsilon^{0}$

$$
u_{i H 0}=0, \quad \tau_{H 0}=\tau_{\mathrm{w}}\left(x_{2}, x_{3}\right)
$$




$$
\begin{array}{cccc} 
& \text { Sone }(2002,2007) & \text { Definition } & \text { Notes } \\
A(\zeta) & A & (2.17 a) & \\
B(\zeta) & B & (2.17 b) & \\
F(\zeta) & F & (2.34) & \\
\varphi_{1}^{(0)}\left(\eta, \zeta_{n}, \zeta\right) & & (2.32 a) & \text { Temperature jump } \\
\varphi_{1}^{(1)}\left(\eta, \zeta_{n}, \zeta\right) & & (2.32 b) & \text { Shear slip } \\
\varphi_{2}^{(1)}\left(\eta, \zeta_{n}, \zeta\right) & & (2.32 b) & \text { Thermal slip } \\
\varphi_{3}^{(1)}\left(\eta, \zeta_{n}, \zeta\right) & & (2.32 b) & \\
c_{1}^{(0)} \varphi_{2}^{(1)}+\varphi_{3}^{(1)} & & & \text { Thermal stress slip } \\
\left(\Omega_{1}^{(0)}(\eta), \Theta_{1}^{(0)}(\eta)\right) & \left(\Omega_{1}, \Theta_{1}\right) & (2.40) & \text { Temperature jump } \\
Y_{1}^{(1)}(\eta) & -Y_{0} & (2.40) & \text { Shear slip } \\
Y_{2}^{(1)}(\eta) & -\frac{1}{2} Y_{1} & (2.40) & \text { Thermal slip } \\
Y_{3}^{(1)}(\eta) & \frac{d_{1}}{2} Y_{1}-Y_{a 4} & (2.40) & \\
c_{1}^{(0)} Y_{2}^{(1)}+Y_{3}^{(1)} & -Y_{a 4} & & \text { Thermal stress slip }
\end{array}
$$

TABLE 2. List of universal functions. The correspondence to the notations in Sone $(2002,2007)$ are also shown.

- Order $\varepsilon^{1}$

$$
\begin{aligned}
& \tau_{H 1}=c_{1}^{(0)} n_{i} \partial_{i} \tau_{H 0}, \\
& u_{1 H 1}=0 \\
& u_{i H 1} t_{i}=b_{1}^{(1)} t_{i} n_{j}\left(\partial_{j} u_{i H 0}+\partial_{i} u_{j H 0}\right)+b_{2}^{(1)} t_{i} \partial_{i} \tau_{H 0} .
\end{aligned}
$$

The formulae for the Knudsen-layer corrections are summarized in (2.39). Table 2 summarizes the universal functions.

\section{Behavior of a slightly rarefied gas between two parallel plates with a discontinuous surface temperature}

In this section, we discuss the case of a discontinuous surface temperature based on the result obtained in the previous section. Let us denote again by $L$ the reference length, by $\rho_{0}$ the reference density, by $T_{0}$ the reference temperature, and by $p_{0}=\rho_{0} R T_{0}$ the reference pressure, and consider the following problem.

\subsection{Problem}

Consider a rarefied gas confined between two parallel plates located at $x_{1}= \pm \pi / 2$, where $L x_{i}$ is the space rectangular coordinate system (Fig. 1). The two plates are heated (or cooled) nonuniformly in the following way. That is, the upper halves $\left(x_{2}>0\right)$ are kept at a uniform temperature $T_{0}\left(1+\tau_{\mathrm{w}}\right)$, while the lower halves $\left(x_{2}<0\right)$ are kept at another uniform temperature $T_{0}\left(1-\tau_{\mathrm{w}}\right)$, where $\tau_{\mathrm{w}}$ is a constant. Thus, the surface temperature of the channel is discontinuous at $x_{2}=0$ with jump $2 T_{0}\left|\tau_{\mathrm{w}}\right|$. There is no pressure gradient imposed on the gas nor external force. Investigate the steady behavior of the gas in the channel on the basis of the Boltzmann equation and the diffuse reflection boundary condition, under the assumption that $\left|\tau_{\mathrm{w}}\right|$ is so small that the equation and boundary conditions can be linearized around the reference equilibrium state at rest. In particular, we pay special attention to the behavior of the gas when the Knudsen number $K n=\ell_{0} / L$, or $\varepsilon=(\sqrt{\pi} / 2) K n$, is small, where $\ell_{0}$ is the mean free path of the gas molecules in the equilibrium state at rest with temperature $T_{0}$ and density $\rho_{0}$. 
On the motion of slightly rarefied gas induced by a discontinuous surface temperature1

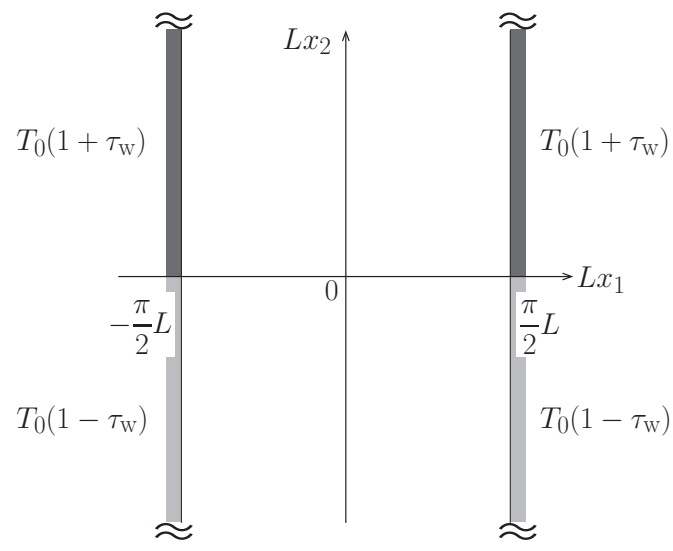

Figure 1. Problem.

We use the same notations as in the previous section. That is, the velocity distribution function is denoted by $\rho_{0}\left(2 R T_{0}\right)^{-3 / 2}\left(1+\phi\left(x_{1}, x_{2}, \zeta_{i}\right)\right) E$. Here, we have assumed that the state of the gas is independent of $x_{3}$. Then, $\phi$ satisfies the following equation and boundary conditions:

$$
\begin{aligned}
& \zeta_{1} \partial_{1} \phi+\zeta_{2} \partial_{2} \phi=\frac{1}{\varepsilon} \mathcal{L}(\phi), \quad\left(-\frac{\pi}{2}<x_{1}<\frac{\pi}{2},-\infty<x_{2}<\infty\right), \\
& \phi=2 \sqrt{\pi} \int_{\zeta_{1}<0}\left|\zeta_{1}\right| \phi E \mathrm{~d} \zeta \pm\left(\zeta_{j}^{2}-2\right) \tau_{\mathrm{w}}, \quad \zeta_{1}>0, \quad\left(x_{1}=-\frac{\pi}{2}, x_{2} \gtrless 0\right), \\
& \phi=2 \sqrt{\pi} \int_{\zeta_{1}>0}\left|\zeta_{1}\right| \phi E \mathrm{~d} \zeta \pm\left(\zeta_{j}^{2}-2\right) \tau_{\mathrm{w}}, \quad \zeta_{1}<0, \quad\left(x_{1}=\frac{\pi}{2}, \quad x_{2} \gtrless 0\right) .
\end{aligned}
$$

The macroscopic variables, the density $\rho_{0}(1+\omega)$, the flow velocity $\left(2 R T_{0}\right)^{1 / 2} u_{i}\left(u_{3}=0\right)$, the temperature $T_{0}(1+\tau)$, and the pressure $p_{0}(1+P)$, are defined as before, and we do not repeat it here (see $(2.4))$. Note that they are functions of $\left(x_{1}, x_{2}\right)$ in the present section.

We observe that a solution that is even in $\left(x_{1}, \zeta_{1}\right)$ and odd (antisymmetric) in $\left(x_{2}, \zeta_{2}\right)$ is compatible with the present problem, i.e.,

$$
\begin{aligned}
& \phi\left(-x_{1}, x_{2},-\zeta_{1}, \zeta_{2}, \zeta_{3}\right)=\phi\left(x_{1}, x_{2}, \zeta_{1}, \zeta_{2}, \zeta_{3}\right), \\
& \phi\left(x_{1},-x_{2}, \zeta_{1},-\zeta_{2}, \zeta_{3}\right)=-\phi\left(x_{1}, x_{2}, \zeta_{1}, \zeta_{2}, \zeta_{3}\right) .
\end{aligned}
$$

Therefore, we may consider the problem in the upper half domain $x_{2}>0$ by imposing the following reflection condition at $x_{2}=0$ :

$$
\phi\left(x_{1}, 0, \zeta_{1}, \zeta_{2}, \zeta_{3}\right)=-\phi\left(x_{1}, 0, \zeta_{1},-\zeta_{2}, \zeta_{3}\right), \quad \zeta_{2}>0 .
$$

In what follows, we denote the restriction of $\phi$ to $x_{2} \gtrless 0$ by $\phi^{ \pm}$and we shall mainly consider $\phi^{+}$in the sequel. Likewise, $h^{ \pm}\left(h=\omega, u_{i}, \tau\right.$, and $\left.P\right)$ denotes the restriction of $h$ to $x_{2} \gtrless 0$. Once $\phi^{+}$is obtained, $\phi^{-}$is readily obtained by the relation $\phi^{-}\left(x_{1}, x_{2}, \zeta_{1}, \zeta_{2}, \zeta_{3}\right)=$ $-\phi^{+}\left(x_{1},-x_{2}, \zeta_{1},-\zeta_{2}, \zeta_{3}\right)$.

\subsection{Asymptotic matching}

We try to investigate the boundary-value problem for $\phi^{+}$for small $\varepsilon \ll 1$. The idea is to apply the method of matched asymptotic expansion (Taguchi 2015; Taguchi \& Suzuki 2017; Dyke 1975) by regarding Sone's asymptotic solution as the outer solution. More precisely, we use the result derived in $\S 2$ to construct the overall solution in the upper 
half domain, which is possible because the temperature of the boundary is uniform. The effect of the discontinuous surface temperature is then transferred to the mismatch of the diffuse reflection conditions (3.2) and (3.3) on $x_{1}=\mp \pi / 2$ and $x_{2}>0$ and the reflection condition (3.5) on $x_{2}=0$ and $-\pi / 2<x_{1}<\pi / 2$ at the corners $\left(x_{1}, x_{2}\right)=(\mp \pi / 2,0)$. This mismatch causes an abrupt spatial variation of the solution in the vicinity of the corner, which Sone's asymptotic solution cannot describe. Thus, we require an internal layer in the vicinity of the corner to alter the Sone's asymptotic solution in a suitable way. The introduction of this new layer (different from the Knudsen layer) is the key ingredient of the present analysis.

In this paper, we shall carry out the asymptotic analysis to the first order of $\varepsilon$.

\subsubsection{Outer solution: zeroth order in $\varepsilon$}

Let us consider the overall solution in the channel, which is intended to describe the behavior of the gas except in the region close to the point of the surface temperature discontinuity. This solution is assumed to have the length scale of variation of the order of unity except in a thin layer adjacent to the boundary (the Knudsen layer), where the solution is allowed to change abruptly in the direction normal to the boundary. We call this solution the outer solution and denote it by attaching the subscript $F$ as $\phi_{F}$ (or $\phi_{F}^{ \pm}$). In this section, we obtain the zeroth order (leading order) approximation of the outer solution $\phi_{F}^{+}$.

First, putting aside the potential difficulty which may arise near the discontinuity points, we express $\phi_{F}^{+}$as the combination of the Hilbert solution $\phi_{H}^{+}$and the Knudsenlayer correction $\phi_{K}^{+}$:

$$
\phi_{F}^{+}=\phi_{H}^{+}+\phi_{K}^{+}
$$

The length scale of variation of the Hilbert solution is of the order of unity, whereas, the Knudsen-layer correction, which is appreciable only in a thin layer adjacent to the boundary, has the length scale of variation of the order of $\varepsilon$ in the direction normal to the boundary (i.e., $\partial_{i} \phi_{H}=O\left(\phi_{H}\right), \partial_{1} \phi_{K}=O\left(\phi_{K} / \varepsilon\right)$, and $\left.\partial_{2} \phi_{K}=O\left(\phi_{K}\right)\right)$. According to $\S 2, \phi_{H}^{+}$and $\phi_{K}^{+}$are expanded in $\varepsilon$ as

$$
\begin{aligned}
& \phi_{H}^{+}=\phi_{H 0}^{+}+\varepsilon \phi_{H 1}^{+}+\cdots, \\
& \phi_{K}^{+}=\varepsilon \phi_{K 1}^{+}+\cdots .
\end{aligned}
$$

Thus, substituting the above expansions into (3.6), we obtain the expansion of the outer solution $\phi_{F}^{+}$in $\varepsilon$ in the form

$$
\phi_{F}^{+}=\phi_{F 0}^{+}+\varepsilon \phi_{F 1}^{+}+\cdots
$$

where

$$
\begin{aligned}
& \phi_{F 0}^{+}=\phi_{H 0}^{+}, \\
& \phi_{F 1}^{+}=\phi_{H 1}^{+}+\phi_{K 1}^{+} .
\end{aligned}
$$

Corresponding to the expansion of $\phi_{F}^{+}$, the macroscopic quantities are also expanded in $\varepsilon$ as

$$
h_{F}^{+}=h_{F 0}^{+}+\varepsilon h_{F 1}^{+}+\cdots, \quad\left(h=\omega, u_{i}, \tau, P\right),
$$

where

$$
\begin{aligned}
& h_{F 0}^{+}=h_{H 0}^{+}, \\
& h_{F 1}^{+}=h_{H 1}^{+}+h_{K 1}^{+} .
\end{aligned}
$$


On the motion of slightly rarefied gas induced by a discontinuous surface temperaturk3

The relation between $h_{H m}^{+}$and $\phi_{H m}^{+}$and that between $h_{K m}^{+}$and $\phi_{K m}^{+}$are given by (2.8) and $(2.38)$ with $\left(\phi_{H m}, h_{H m}\right)=\left(\phi_{H m}^{+}, h_{H m}^{+}\right)$and $\left(\phi_{K m}, h_{K m}\right)=\left(\phi_{K m}^{+}, h_{K m}^{+}\right)$, respectively.

From the result of $\S 2$, the leading-order term of the outer solution $\phi_{F 0}^{+}$is given by the following local Maxwellian:

$$
\phi_{F 0}^{+}=\phi_{H 0}^{+}=P_{H 0}^{+}+2 \zeta_{1} u_{1 H 0}^{+}+2 \zeta_{2} u_{2 H 0}^{+}+\left(\zeta_{j}^{2}-\frac{5}{2}\right) \tau_{H 0}^{+},
$$

where the macroscopic variables solve the following Stokes problem in $D^{+}=\left\{\left(x_{1}, x_{2}\right) \mid-\right.$ $\left.\pi / 2<x_{1}<\pi / 2, x_{2}>0\right\}$ :

$$
\begin{aligned}
& \partial_{i} P_{H 0}^{+}=0, \\
& \partial_{i} u_{i H 0}^{+}=0, \quad \gamma_{1} \nabla^{2} u_{i H 0}^{+}-\partial_{i} P_{H 1}^{+}=0, \\
& \nabla^{2} \tau_{H 0}^{+}=0,
\end{aligned}
$$

with the boundary conditions

$$
\begin{array}{ll}
u_{i H 0}^{+}=0, & \left(x_{1}=\mp \frac{\pi}{2}, x_{2}>0\right), \\
\tau_{H 0}^{+}=\tau_{\mathrm{w}}, & \left(x_{1}=\mp \frac{\pi}{2}, x_{2}>0\right),
\end{array}
$$

supplemented by the following condition at $x_{2}=0$, i.e.,

$$
P_{H 0}^{+}=u_{1 H 0}^{+}=\tau_{H 0}^{+}=\partial_{2} u_{2 H 0}^{+}=0, \quad\left(-\frac{\pi}{2}<x_{1}<\frac{\pi}{2}, x_{2}=0\right),
$$

(see (3.5)). Here, $i \in\{1,2\}$ and $\nabla^{2}=\partial_{1}^{2}+\partial_{2}^{2}$ (the two-dimensional Laplacian). Equations (3.14a) and (3.14b) are the no-slip/no-jump conditions for the flow velocity and temperature, respectively (see (2.44)). Note that the density is determined from the temperature and pressure by the relation $\omega_{H 0}^{+}=P_{H 0}^{+}-\tau_{H 0}^{+}$.

First,

$$
P_{H 0}^{+}=0,
$$

trivially satisfies $(3.13 a)$ and $(3.15)$. Next, it is readily seen that

$$
u_{i H 0}^{+}=0, \quad P_{H 1}^{+}=0,
$$

is also a trivial solution to the Stokes equations (3.13b) that satisfies the boundary conditions $(3.14 a)$ and (3.15). Note that $P_{H 1}^{+}$is only determined up to an additive constant from the Stokes equation. This constant, which should be determined from the information on the higher order, turns out to be zero if one considers the requirement (3.5). This result has already been used in (3.17). On the other hand, $\tau_{H 0}^{+}$satisfying the Laplace equation $(3.13 c)$ and the boundary conditions (3.14b) and (3.15) is given by

$$
\begin{aligned}
& \tau_{H 0}^{+}=\frac{\tau_{\mathrm{w}}}{\pi} \operatorname{Arg}\left(\frac{1+\sin z}{1-\sin z}\right), \\
& z=x_{1}+\mathrm{i} x_{2},
\end{aligned}
$$

where $\mathrm{i}$ is the imaginary unit. To summarize, the leading-order term of the outer solution is obtained as

$$
\phi_{F 0}^{+}=\left(\zeta_{j}^{2}-\frac{5}{2}\right) \tau_{H 0}^{+},
$$

with $\tau_{H 0}^{+}$given by $(3.18)$. 
We observe that $\tau_{H 0}^{+}$takes different values depending on the way of approach to the point $\left(x_{1}, x_{2}\right)=(-\pi / 2,0)$ or $(\pi / 2,0)$. This is seen by introducing a local polar coordinate system $(r, \theta)$ centered at $\left(x_{1}, x_{2}\right)=(\mp \pi / 2,0)$ by

$$
x_{1}=\mp \frac{\pi}{2} \pm r \cos \theta, \quad x_{2}=r \sin \theta, \quad\left(\text { near }\left(x_{1}, x_{2}\right)=\left(\mp \frac{\pi}{2}, 0\right)\right),
$$

$(0 \leqslant \theta \leqslant \pi / 2)$. Expanding $\tau_{H 0}^{+}$near each of the discontinuity points in terms of small $r$, we obtain

$$
\frac{\tau_{H 0}^{+}}{\tau_{\mathrm{w}}}=\frac{2}{\pi} \theta+\frac{r^{2}}{6 \pi} \sin 2 \theta+O\left(r^{4}\right), \quad(r \ll 1) .
$$

This shows that the limiting value of $\tau_{H 0}^{+}$and therefore that of $\phi_{F 0}^{+}$as $r \searrow 0$ are not uniquely determined but depend on $\theta$.

\subsubsection{Outer solution: first order in $\varepsilon$}

Next, we proceed to the first order in the expansion of the outer solution. Unlike the zeroth order, $\phi_{F 1}^{+}$is composed of the Hilbert part $\phi_{H 1}^{+}$and the Knudsen-layer part $\phi_{K 1}^{+}($see $(3.9 b))$. More precisely, with (3.18) and (3.17) obtained above, (2.43) with $\partial_{3}=u_{3 H 1}=0$ is computed to give

$$
\begin{aligned}
& \phi_{H 1}^{+}=2 \zeta_{1} u_{1 H 1}^{+}+2 \zeta_{2} u_{2 H 1}^{+}+\left(\zeta_{j}^{2}-\frac{5}{2}\right) \tau_{H 1}^{+}-\frac{4 \tau_{\mathrm{w}}}{\pi} A(\zeta) \frac{\zeta_{1} \sin x_{1} \sinh x_{2}+\zeta_{2} \cos x_{1} \cosh x_{2}}{\cos \left(2 x_{1}\right)+\cosh \left(2 x_{2}\right)} \\
& \phi_{K 1}^{+}=-\frac{2 \tau_{\mathrm{w}}}{\pi} \frac{1}{\sinh x_{2}}\left(\varphi_{1}^{(0)}\left(\frac{\left|x_{1}+\frac{\pi}{2}\right|}{\varepsilon}, \zeta_{1}, \zeta\right)+\varphi_{1}^{(0)}\left(\frac{\left|x_{1}-\frac{\pi}{2}\right|}{\varepsilon},-\zeta_{1}, \zeta\right)\right),
\end{aligned}
$$

where $\zeta=\left(\zeta_{j}^{2}\right)^{1 / 2}$ and $A$ and $\varphi_{1}^{(0)}$ are the functions defined in $(2.17 a)$ and $(2.32 a)$, respectively. Note that $\varphi_{1}^{(0)}$ is meaningful only in the Knudsen layer. Therefore, there is no overlap of the two terms in $(3.22 b)$. The quantities $u_{i H 1}^{+}=u_{i H 1}^{+}\left(x_{1}, x_{2}\right)$ and $\tau_{H 1}^{+}=$ $\tau_{H 1}^{+}\left(x_{1}, x_{2}\right)$ contained in $(3.22 a)$ are determined by solving the first-order Stokes problem. We will present the explicit form of the problem later (see (3.37)-(3.39)). For the moment, it suffices to mention that, on solving this problem, we simply obtain the following firstorder macroscopic quantities with a trivial flow velocity:

$$
\begin{aligned}
& \tau_{H 1}^{+}=-\omega_{H 1}^{+}=-\frac{8 \tau_{\mathrm{w}} c_{1}^{(0)}}{\pi^{2}} \frac{x_{2} \cos x_{1} \cosh x_{2}+x_{1} \sin x_{1} \sinh x_{2}}{\cos \left(2 x_{1}\right)+\cosh \left(2 x_{2}\right)}, \\
& u_{1 H 1}^{+}=u_{2 H 1}^{+}=0, \quad P_{H 2}^{+}=\text {const. }
\end{aligned}
$$

Note that the trivial flow velocity is the consequence of the no-slip boundary condition $u_{i H 1}=0$ on the boundary, which results from (2.45) because $\tau_{\mathrm{w}}$ is constant and $u_{i H 0}=0$.

Of course, our interest is a nontrivial flow velocity and the above result needs to be modified in a suitable way. At this point, however, we simply examine how $\phi_{F 1}^{+}$thus obtained (denoted by $\phi_{F 1 *}^{+}$) behaves near the points of discontinuity.

Using again the local polar coordinates introduced in (3.20) as well as the corresponding circumferential molecular velocity $\zeta_{\theta}=\mp \zeta_{1} \sin \theta+\zeta_{2} \cos \theta$ near $\left(x_{1}, x_{2}\right)=(\mp \pi / 2,0)$, the essential terms of $\phi_{F 1 *}^{+}=\phi_{H 1}^{+}+\phi_{K 1}^{+}$(with (3.22) and (3.23)) in the vicinity of $\left(x_{1}, x_{2}\right)=(\mp \pi / 2,0)$ takes the form

$$
\begin{aligned}
\phi_{F 1 *}^{+} \simeq & -\frac{2 \tau_{\mathrm{w}}}{\pi}\left[\frac{c_{1}^{(0)} \sin \theta}{r}\left(\zeta_{j}^{2}-\frac{5}{2}\right)+\frac{\zeta_{\theta}}{r} A(\zeta)+\frac{1}{x_{2}} \varphi_{1}^{(0)}\left(\frac{\left|x_{1} \pm \frac{\pi}{2}\right|}{\varepsilon}, \pm \zeta_{1}, \zeta\right)\right] \\
& +O(r), \quad(r \ll 1) .
\end{aligned}
$$


On the motion of slightly rarefied gas induced by a discontinuous surface temperaturk5

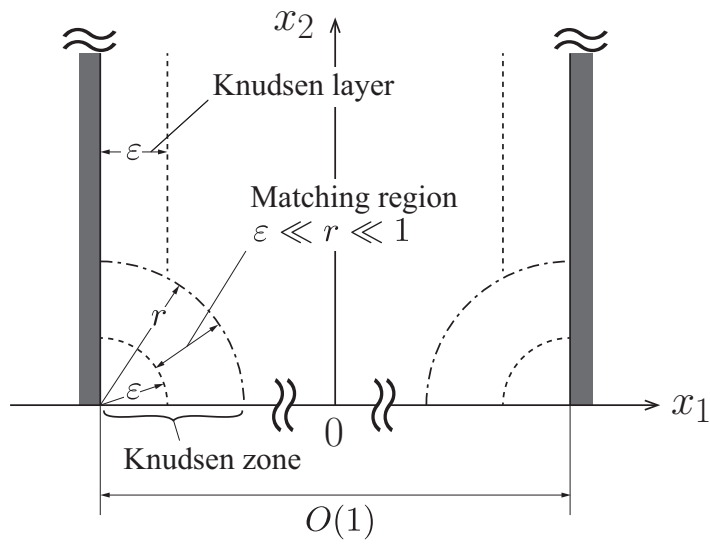

FIGURE 2. Schematics of the solution structure. Only the upper half domain is shown.

Thus, $\left|\phi_{F 1 *}^{+}\right|$diverges with the rate $r^{-1}$ on approaching the points of discontinuity. In other words, the outer solution based on Sone's asymptotic theory is valid only in the region $r \gg \varepsilon$.

\subsubsection{Inner solution: zeroth order (leading order) in $\varepsilon$}

We have seen that the outer solution breaks down in the vicinity of the point where the surface temperature has a jump. The reason is that the underlying length scale of variation of the Hilbert solution and that of the Knudsen-layer correction are too restrictive to describe the abrupt change that the flow undergoes near the points of discontinuity. This motivates us to introduce another inner layer near $\left(x_{1}, x_{2}\right)=(\mp \pi / 2,0)$, in which the solution is allowed to vary in $x_{1}$ and $x_{2}$ on the length scale of variation of the order of the (scaled) mean free path. This localized region near each of the discontinuity points may be called the Knudsen zone (see Fig. 2). Accordingly, the solution in this region is hereafter denoted by $\phi=\phi_{Z}$, i.e., $\partial_{i} \phi_{Z}=O\left(\phi_{Z} / \varepsilon\right)$.

In order to analyze the Knudsen zone, it is convenient to introduce new variables $y_{i}$ (the inner variables) and $\xi_{i}$ by the relations

$$
x_{1}=\mp \frac{\pi}{2} \pm \varepsilon y_{1}, \quad x_{2}=\varepsilon y_{2}, \quad\left(\xi_{1}, \xi_{2}, \xi_{3}\right)=\left( \pm \zeta_{1}, \zeta_{2}, \zeta_{3}\right), \quad \text { near }\left(x_{1}, x_{2}\right)=\left(\mp \frac{\pi}{2}, 0\right),
$$

and express the solution as $\phi_{Z}=\phi_{Z}\left(y_{1}, y_{2}, \xi_{i}\right)$. If we further expand $\phi_{Z}$ in a power series of $\varepsilon$, i.e.,

$$
\phi_{Z}=\phi_{Z 0}+\varepsilon \phi_{Z 1}+\cdots
$$

the leading-order term $\phi_{Z 0}$ satisfies the following equation and boundary condition:

$$
\begin{aligned}
& \xi_{1} \frac{\partial \phi_{Z 0}}{\partial y_{1}}+\xi_{2} \frac{\partial \phi_{Z 0}}{\partial y_{2}}=\mathcal{L}\left(\phi_{Z 0}\right), \quad\left(y_{1}>0,-\infty<y_{2}<\infty\right), \\
& \phi_{Z 0}=2 \sqrt{\pi} \int_{\xi_{1}<0}\left|\xi_{1}\right| \phi_{Z 0} \operatorname{Ed} \boldsymbol{\xi} \pm\left(\xi_{j}^{2}-2\right) \tau_{\mathrm{w}}, \quad \xi_{1}>0, \quad\left(y_{1}=0, \pm y_{2}>0\right),
\end{aligned}
$$

where $\mathbf{E}=E\left(\xi_{i}\right)$ and $\mathrm{d} \boldsymbol{\xi}=\mathrm{d} \xi_{1} \mathrm{~d} \xi_{2} \mathrm{~d} \xi_{3}$.

To derive the boundary condition at infinity, we use the asymptotic matching. Let us consider a point $\left(x_{1}, x_{2}\right)$ close to the discontinuity point that satisfies $r=\sqrt{\left(x_{1} \pm \pi / 2\right)^{2}+x_{2}^{2}} \ll 1$ and $r \gg \varepsilon$. As discussed above, the outer solution is 
valid in the region $r \gg \varepsilon$. If we further assume that the inner solution is valid in the region $r \ll 1$, there appears a crossover of the two solutions in the region $\varepsilon \ll r \ll 1$. With this kept in mind, we express the zeroth-order outer solution $\phi_{F 0}=\phi_{F 0}\left(x_{1}, x_{2}, \zeta_{i}\right)$ in terms of the inner variables $\left(y_{1}, y_{2}, \xi_{i}\right)$. As the result, we obtain the following asymptotic expression of the outer solution $\phi_{F}=\phi_{F 0}+\varepsilon \phi_{F 1}+\cdots$ in terms of $\varepsilon$ in the vicinity of $\left(x_{1}, x_{2}\right)=(\mp \pi / 2,0)$ :

$$
\phi_{F}^{\dagger}=\frac{2 \tau_{w}}{\pi}\left(\xi_{j}^{2}-\frac{5}{2}\right) \theta+O(\varepsilon), \quad(\text { as } \varepsilon \searrow 0 \text { with } r=\varepsilon \widetilde{r} \text { fixed }),
$$

where $\theta=\operatorname{Arctan}\left(y_{2} / y_{1}\right), \widetilde{r}=\sqrt{y_{1}^{2}+y_{2}^{2}}$ and the superscript $\dagger$ is attached to emphasize that the outer solution is expressed in terms of the inner variables. Note that the first term is independent of $\widetilde{r}$, because so is the first term of $(3.21)$, but the $O(\varepsilon)$ term is generally $\widetilde{r}$-dependent. Therefore, in order for the inner solution $\phi_{Z}$ to match $\phi_{F}$ disregarding the error of $O(\varepsilon)$ as $\varepsilon \searrow 0$, we require

$$
\phi_{Z 0} \rightarrow \frac{2 \tau_{w}}{\pi}\left(\xi_{j}^{2}-\frac{5}{2}\right) \theta, \quad(\widetilde{r} \rightarrow \infty) .
$$

Finally, the macroscopic quantities in the Knudsen zone (at the zeroth order) are expressed in terms of $\phi_{Z 0}$ as follows:

$$
\begin{aligned}
\omega_{Z 0} & =\int \phi_{Z 0} \mathrm{Ed} \boldsymbol{\xi}, \quad u_{i Z 0}=\int \xi_{i} \phi_{Z 0} \mathrm{Ed} \boldsymbol{\xi}, \quad(i=1,2), \quad u_{3 Z 0}=0, \\
\tau_{Z 0} & =\frac{2}{3} \int\left(\xi_{j}^{2}-\frac{3}{2}\right) \phi_{Z 0} \mathrm{Ed} \boldsymbol{\xi}, \quad P_{Z 0}=\omega_{Z 0}+\tau_{Z 0},
\end{aligned}
$$

where the integration is carried out over the whole $\xi_{i}$ space.

Equations (3.27), (3.28), and (3.30) form a (spatially two-dimensional) boundary-value problem of the linearized Boltzmann equation for $\phi_{z 0}$ in a half space. Physically, the problem describes the steady motion of a rarefied gas caused by a discontinuous surface temperature over plane boundary in the situation where there is a steady heat flow (flowing in the clockwise direction when $\tau_{\mathrm{w}}>0$ ) at a far distance. As we will see, this problem admits a solution with nonzero flow velocity whose magnitude is inversely proportional to the distance from the origin in the far field. From this behavior at far field, a condition for $u_{i H 1}$ of the Hilbert solution is derived.

We may assume that the solution is antisymmetric with respect to $\left(y_{2}, \xi_{2}\right)$, i.e.,

$$
\phi_{Z 0}\left(y_{1},-y_{2}, \xi_{1},-\xi_{2}, \xi_{3}\right)=-\phi_{Z 0}\left(y_{1}, y_{2}, \xi_{1}, \xi_{2}, \xi_{3}\right) .
$$

Therefore, we only need to solve the problem in the upper half domain $y_{2}>0$, by imposing the following reflection condition at $y_{2}=0$, i.e.,

$$
\phi_{Z 0}\left(y_{1}, 0, \xi_{1},-\xi_{2}, \xi_{3}\right)=-\phi_{Z 0}\left(y_{1}, 0, \xi_{1}, \xi_{2}, \xi_{3}\right), \quad \xi_{2}>0 .
$$

\subsubsection{Far-field behavior of the gas in the Knudsen zone}

This subsection is devoted to a discussion of the asymptotic behavior of the solution in the far field $\left(\widetilde{r}=\sqrt{y_{1}^{2}+y_{2}^{2}} \gg 1\right)$ of the Knudsen-zone problem. The key observation is that, in the far field, the length scale of variation of the solution is large (i.e., the local Knudsen numbers is small), and one can apply Sone's asymptotic method (the linear theory (Sone 2007, Chap. 3)). The application of the theory is straightforward and the derivation is outlined in Appendix A. Here, we list the resulting asymptotic forms of the macroscopic quantities as well as that of the velocity distribution function in the far 
On the motion of slightly rarefied gas induced by a discontinuous surface temperaturk7 region $\widetilde{r} \gg 1$.

a. macroscopic quantities

$$
\begin{aligned}
\frac{u_{r Z 0}}{\tau_{\mathrm{w}}}= & \frac{\Gamma_{Z}^{(1)}}{\widetilde{r}} \sin 2 \theta-\frac{3 \Gamma_{Z}^{(2)}(\sin 3 \theta+\sin \theta)-2\left(\Gamma_{Z}^{(1)} b_{1}^{(1)}+\frac{c_{1}^{(0)} b_{2}^{(1)}+b_{3}^{(1)}}{\pi}\right) \sin \theta}{\widetilde{r}^{2}} \\
& +\frac{2}{y_{2}^{2}}\left(\Gamma_{Z}^{(1)} Y_{1}^{(1)}\left(y_{1}\right)+\frac{c_{1}^{(0)} Y_{2}^{(1)}\left(y_{1}\right)+Y_{3}^{(1)}\left(y_{1}\right)}{\pi}\right) \sin \theta+\cdots, \\
\frac{u_{\theta Z}}{\tau_{\mathrm{w}}} & =\frac{\Gamma_{Z}^{(2)}(\cos 3 \theta+3 \cos \theta)-2\left(\Gamma_{Z}^{(1)} b_{1}^{(1)}+\frac{c_{1}^{(0)} b_{2}^{(1)}+b_{3}^{(1)}}{\pi}\right) \cos \theta}{\widetilde{r}^{2}} \\
& +\frac{2}{y_{2}^{2}}\left(\Gamma_{Z}^{(1)} Y_{1}^{(1)}\left(y_{1}\right)+\frac{c_{1}^{(0)} Y_{2}^{(1)}\left(y_{1}\right)+Y_{3}^{(1)}\left(y_{1}\right)}{\pi}\right) \cos \theta+\cdots, \\
\frac{P_{Z 0}}{\tau_{\mathrm{w}}} & =-\frac{2}{\pi} \frac{1}{y_{2}}\left(\Omega_{1}^{(0)}\left(y_{1}\right)+\Theta_{1}^{(0)}\left(y_{1}\right)\right)+\frac{2 \gamma_{1} \Gamma_{Z}^{(1)}}{\widetilde{r}^{2}} \sin 2 \theta+\cdots, \\
\frac{\tau_{Z 0}}{\tau_{\mathrm{w}}} & =\frac{2}{\pi} \theta-\frac{2}{\pi}\left(\frac{c_{1}^{(0)}}{\widetilde{r}} \sin \theta+\frac{1}{y_{2}} \Theta_{1}^{(0)}\left(y_{1}\right)\right)+\frac{\Lambda_{Z}^{(2)}}{\widetilde{r}^{2}} \sin 2 \theta+\cdots, \\
\frac{\omega_{Z 0}}{\tau_{\mathrm{w}}} & =-\frac{2}{\pi} \theta+\frac{2}{\pi}\left(\frac{c_{1}^{(0)}}{\widetilde{r}} \sin \theta-\frac{1}{y_{2}} \Omega_{1}^{(0)}\left(y_{1}\right)\right)+\frac{2 \gamma_{1} \Gamma_{Z}^{(1)}-\Lambda_{Z}^{(2)}}{\widetilde{r}^{2}} \sin 2 \theta+\cdots
\end{aligned}
$$

b. velocity distribution function

$$
\begin{aligned}
\frac{\phi_{Z 0}}{\tau_{\mathrm{w}}} & =\frac{2 \Gamma_{Z}^{(1)}}{\widetilde{r}} \xi_{r} \sin 2 \theta+\frac{2}{\pi}\left(\xi_{j}^{2}-\frac{5}{2}\right)\left(\theta-\frac{c_{1}^{(0)}}{\widetilde{r}} \sin \theta+\frac{\pi}{2} \frac{\Lambda_{Z}^{(2)}}{\widetilde{r}^{2}} \sin 2 \theta\right) \\
& -\frac{2}{\pi}\left(\frac{\xi_{\theta}}{\widetilde{r}} A(\xi)+\frac{1}{y_{2}} \varphi_{1}^{(0)}\left(y_{1}, \xi_{1}, \xi\right)\right)+\frac{2 \gamma_{1} \Gamma_{Z}^{(1)}}{\widetilde{r}^{2}} \sin 2 \theta \\
& -2 \Gamma_{Z}^{(2)} \frac{3 \xi_{r}(\sin 3 \theta+\sin \theta)-\xi_{\theta}(\cos 3 \theta+3 \cos \theta)}{\widetilde{r}^{2}} \\
& +4\left(\Gamma_{Z}^{(1)} b_{1}^{(1)}+\frac{c_{1}^{(0)} b_{2}^{(1)}+b_{3}^{(1)}}{\pi}\right) \frac{\xi_{r} \sin \theta-\xi_{\theta} \cos \theta}{\widetilde{r}^{2}} \\
& +\Gamma_{Z}^{(1)}\left(\frac{\left(\xi_{r}^{2}-\xi_{\theta}^{2}\right) \sin 2 \theta-2 \xi_{r} \xi_{\theta} \cos 2 \theta}{\widetilde{r}^{2}} B(\xi)+\frac{2 \xi_{2}}{y_{2}^{2}} \varphi_{1}^{(1)}\left(y_{1}, \xi_{1}, \xi\right)\right) \\
& +\frac{2}{\pi} c_{1}^{(0)}\left(-\frac{\xi_{r} \sin \theta-\xi_{\theta} \cos \theta}{\widetilde{r}^{2}} A(\xi)+\frac{\xi_{2}}{y_{2}^{2}} \varphi_{2}^{(1)}\left(y_{1}, \xi_{1}, \xi\right)\right) \\
& +\frac{2}{\pi}\left(\frac{2 \xi_{r} \xi_{\theta}}{\widetilde{r}^{2}} F(\xi)+\frac{\xi_{2}}{y_{2}^{2}} \varphi_{3}^{(1)}\left(y_{1}, \xi_{1}, \xi\right)\right)+\cdots
\end{aligned}
$$

Here, $\Gamma_{Z}^{(1)}, \Gamma_{Z}^{(2)}$, and $\Lambda_{Z}^{(2)}$ are constants which should be determined together with the solution, $\xi=\left(\xi_{j}^{2}\right)^{1 / 2}=\left(\xi_{r}^{2}+\xi_{\theta}^{2}+\xi_{3}^{2}\right)^{1 / 2}$,

$$
\left(\begin{array}{ll}
\xi_{r} & u_{r Z 0} \\
\xi_{\theta} & u_{\theta Z 0}
\end{array}\right)=\left(\begin{array}{cc}
\cos \theta & \sin \theta \\
-\sin \theta & \cos \theta
\end{array}\right)\left(\begin{array}{ll}
\xi_{1} & u_{1 Z 0} \\
\xi_{2} & u_{2 Z 0}
\end{array}\right),
$$

and the constants $\left(\gamma_{1}, c_{1}^{(0)}, b_{j}^{(1)}\right)$ and the functions $\left(A, B, \varphi_{1}^{(0)}, \varphi_{j}^{(1)}, \Omega_{1}^{(0)}, \Theta_{1}^{(0)}, Y_{j}^{(1)}\right)$ are the same as those introduced in $\S 2$ (see Tables 1 and 2 ). 
As seen from (3.34), the flow velocity has radially diverging or converging streamlines depending on $y_{1} \gtrless 0$ in the far field, provided that $\Gamma_{Z}^{(1)}$ is positive. Our preliminary numerical analysis employing the BGK model shows that this is likely to hold, which is also consistent with the numerical results shown later in $\S 4$.

The Knudsen-zone problem is spatially two-dimensional, and the numerical analysis is much more difficult than the conventional Knudsen-layer problem, which is spatially one dimensional. Therefore, we shall present the numerical result of the Knudsen-zone problem in detail in a separate paper. For the moment, we assume that $\Gamma_{Z}^{(1)}, \Gamma_{Z}^{(2)}$, and $\Lambda_{Z}^{(2)}$ are known and proceed to the next step of the asymptotic analysis.

\subsubsection{Outer solution: first order in $\varepsilon$ and a diverging singularity to drive the flow}

We go back to the first-order outer solution and consider $\phi_{F 1}^{+}$. Applying the results of $\S 2$, the overall behavior of the macroscopic quantities in the outer region are described by the following Stokes problem:

a. equations

$$
\begin{aligned}
& \partial_{i} u_{i H 1}^{+}=0, \quad \gamma_{1} \nabla^{2} u_{i H 1}^{+}-\partial_{i} P_{H 2}^{+}=0, \\
& \nabla^{2} \tau_{H 1}^{+}=0
\end{aligned}
$$

\section{b. boundary conditions}

$$
\begin{aligned}
& u_{i H 1}^{+}=0, \quad\left(x_{1}=\mp \frac{\pi}{2}, x_{2}>0\right), \\
& \tau_{H 1}^{+}= \pm c_{1}^{(0)} \partial_{1} \tau_{H 0}^{+}=-\frac{2 \tau_{\mathrm{w}} c_{1}^{(0)}}{\pi} \frac{1}{\sinh x_{2}}, \quad\left(x_{1}=\mp \frac{\pi}{2}, x_{2}>0\right),
\end{aligned}
$$

The conditions (3.38a) and (3.38b) correspond to the no-slip condition for the flow velocity $u_{i H 1}^{+}$and to the jump condition for the temperature $\tau_{H 1}^{+}$, respectively (see (2.45)). Note that there is no thermal slip because $\tau_{H 0}^{+}$is constant along the boundary $x_{1}=\mp \pi / 2$. In addition, we impose the following condition at $x_{2}=0$ :

$$
u_{1 H 1}^{+}=\tau_{H 1}^{+}=\partial_{2} u_{2 H 1}^{+}=0, \quad\left(-\frac{\pi}{2}<x_{1}<\frac{\pi}{2}, x_{2}=0\right) .
$$

The first order density is obtained from the temperature by $\omega_{H 1}^{+}=-\tau_{H 1}^{+}$since $P_{H 1}^{+}=0$.

Solving the problem for $\tau_{H 1}^{+}$, we obtain the temperature $\tau_{H 1}^{+}$announced in $(3.23 a)$. On the other hand, (3.37a) with (3.38a) and (3.39) yields merely a trivial flow field $(3.23 b)$, which is not of our interest. However, the difference from $\S 3.2 .2$ is that we have now the Knudsen zone as a part of the solution; a condition for nontrivial flow is obtained by the consideration that the outer solution should match asymptotically the inner solution in the crossover region.

With the same notation as in (3.20), let us consider a point close to $\left(x_{1}, x_{2}\right)=(\mp \pi / 2,0)$ for which $\varepsilon \ll r \ll 1$. Note that this range corresponds to $1 \ll \widetilde{r} \ll \varepsilon^{-1}$ in the the inner region. Therefore, for the corresponding inner solution, we can use the far-field asymptotic form (3.35) for the leading-order Knudsen-zone solution $\phi_{Z 0}$. With the aid of the relation between the inner and outer variables (i.e., (3.25)), this is further transformed into the 
On the motion of slightly rarefied gas induced by a discontinuous surface temperaturk9 expansion in $\varepsilon$ as follows:

$$
\begin{aligned}
\frac{\phi_{Z 0}^{\dagger}}{\tau_{\mathrm{w}}} & =\varepsilon \frac{2 \Gamma_{Z}^{(1)}}{r} \zeta_{r} \sin 2 \theta+\frac{2}{\pi}\left(\zeta_{j}^{2}-\frac{5}{2}\right)\left(\theta-\varepsilon \frac{c_{1}^{(0)}}{r} \sin \theta\right) \\
& -\varepsilon \frac{2}{\pi}\left(\frac{\zeta_{\theta}}{r} A(\zeta)+\frac{1}{x_{2}} \varphi_{1}^{(0)}\left(\frac{\left|x_{1} \pm \frac{\pi}{2}\right|}{\varepsilon}, \pm \zeta_{1}, \zeta\right)\right)+O\left(\varepsilon^{2}\right), \\
& =\frac{2}{\pi}\left(\zeta_{j}^{2}-\frac{5}{2}\right) \theta+\varepsilon\left[\frac{2 \Gamma_{Z}^{(1)}}{r} \zeta_{r} \sin 2 \theta-\frac{2}{\pi} \frac{c_{1}^{(0)}}{r} \sin \theta\left(\zeta_{j}^{2}-\frac{5}{2}\right)\right. \\
& \left.-\frac{2}{\pi} \frac{\zeta_{\theta}}{r} A(\zeta)-\frac{2}{\pi} \frac{1}{x_{2}} \varphi_{1}^{(0)}\left(\frac{\left|x_{1} \pm \frac{\pi}{2}\right|}{\varepsilon}, \pm \zeta_{1}, \zeta\right)\right]+O\left(\varepsilon^{2}\right), \quad \text { as } \varepsilon \searrow 0 \text { with } \widetilde{r}=\frac{r}{\varepsilon} \text { fixed, }
\end{aligned}
$$

where the superscript $\dagger$ is attached to indicate that the function is expressed in terms of the outer variables $\left(x_{i}, \zeta_{i}\right)$. On the other hand, (3.19), (3.22a) with $(3.23 a)$, and $(3.22 b)$ give, for small $r$,

$$
\begin{aligned}
\phi_{F}^{+} & =\phi_{F 0}^{+}+\varepsilon \phi_{F 1}^{+}+\cdots \\
& =\frac{2 \tau_{\mathrm{w}}}{\pi}\left(\zeta_{j}^{2}-\frac{5}{2}\right)\left(\theta+O\left(r^{2}\right)\right) \\
& +\varepsilon\left[2\left(\zeta_{r} u_{r H 1}^{+}+\zeta_{\theta} u_{\theta H 1}^{+}\right)-\frac{2 \tau_{\mathrm{w}}}{\pi} \frac{c_{1}^{(0)}}{r} \sin \theta\left(\zeta_{j}^{2}-\frac{5}{2}\right)\right. \\
& \left.-\frac{2 \tau_{\mathrm{w}}}{\pi} \frac{\zeta_{\theta}}{r} A(\zeta)-\frac{2 \tau_{\mathrm{w}}}{\pi} \frac{1}{x_{2}} \varphi_{1}^{(0)}\left(\frac{\left|x_{1} \pm \frac{\pi}{2}\right|}{\varepsilon}, \pm \zeta_{1}, \zeta\right)+O(r)\right]+\cdots
\end{aligned}
$$

Therefore, the comparison of the the $\varepsilon$ order terms in the two expressions shows that the macroscopic flow velocity $u_{r H 1}^{+}$and $u_{\theta H 1}^{+}$appearing in (3.41) must satisfy the following matching conditions as $r \searrow 0$ :

$$
u_{r H 1}^{+} \rightarrow \frac{\tau_{\mathrm{w}} \Gamma_{Z}^{(1)}}{r} \sin 2 \theta, \quad u_{\theta H 1}^{+} \rightarrow 0, \quad(r \rightarrow 0) .
$$

The condition (3.42) plays the role of a driving force for the flow caused by a discontinuous surface temperature on a planar surface, and its magnitude $\Gamma_{Z}^{(1)}$ is determined through the analysis of the Knudsen-zone problem (or two-dimensional Knudsen-layer problem). In summary, the flow field at the first order in $\varepsilon$ is determined by solving the Stokes equation (3.37a) under the boundary conditions (3.38a), (3.39) and the matching condition (3.42).

\subsection{Summary of the fluid-dynamic-type system}

We summarize the result of the asymptotic analysis carried out in this section. Outside the Knudsen zone near the discontinuity point, the macroscopic variables are expressed as

$$
\begin{aligned}
& u_{i}=\varepsilon u_{i H 1}+O\left(\varepsilon^{2}\right), \quad P=\varepsilon P_{K 1}+O\left(\varepsilon^{2}\right), \\
& \tau=\tau_{H 0}+\varepsilon\left(\tau_{H 1}+\tau_{K 1}\right)+O\left(\varepsilon^{2}\right), \\
& \omega=\omega_{H 0}+\varepsilon\left(\omega_{H 1}+\omega_{K 1}\right)+O\left(\varepsilon^{2}\right),
\end{aligned}
$$

where $\tau_{H 0}$ is given by $(3.18)$, and $\tau_{H 1}$ and $u_{i H 1}\left(u_{3 H 1}=0\right)$ are determined by solving the following Stokes problem: 
a. equations

$$
\begin{aligned}
\partial_{i} u_{i H 1} & =0, \quad \gamma_{1} \nabla^{2} u_{i H 1}-\partial_{i} P_{H 2}=0, \\
\nabla^{2} \tau_{H 1} & =0
\end{aligned}
$$

b. boundary conditions

$$
\begin{aligned}
& u_{i H 1}=0, \quad\left(x_{1}=\mp \frac{\pi}{2}, x_{2} \neq 0\right), \\
& \tau_{H 1}= \pm c_{1}^{(0)} \partial_{1} \tau_{H 0}, \quad\left(x_{1}=\mp \frac{\pi}{2}, x_{2} \neq 0\right)
\end{aligned}
$$

c. matching conditions

$$
u_{r H 1} \rightarrow \frac{\tau_{\mathrm{w}} \Gamma_{Z}^{(1)}}{r} \sin 2 \theta, \quad u_{\theta H 1} \rightarrow 0, \quad\left(x_{1}, x_{2}\right) \rightarrow\left(\mp \frac{\pi}{2}, 0\right) .
$$

Here, $(r, \theta)(-\pi / 2 \leqslant \theta \leqslant \pi / 2)$ is the polar coordinate system centered at $\left(x_{1}, x_{2}\right)=$ $(\mp \pi / 2,0)$, i.e., $x_{1}=\mp \pi / 2 \pm r \cos \theta$ and $x_{2}=r \sin \theta$. The density $\omega$ up to the first order in $\varepsilon$ is related to the temperature as $\omega_{H 0}=-\tau_{H 0}$ and $\omega_{H 1}=-\tau_{H 1}$ since $P_{H}=O\left(\varepsilon^{2}\right)$.

In this paper, we will not give the solution to the above problem for $u_{i H 1}$ and $P_{H 2}\left(\tau_{H 1}\right.$ and $\omega_{H 1}$ are given in $(3.23 a)$ ). Instead, we verify whether the diverging condition (3.46) is actually observed around a flat boundary with a discontinuous surface temperature in a direct numerical computation.

We close this section with the following comments. In this study, we used the linearized Boltzmann equation. The linearization is legitimate as long as the jump $2 T_{0}\left|\tau_{\mathrm{w}}\right|$ in the surface temperature is sufficiently small compared with the reference temperature $T_{0}$. There is no restriction imposed on the magnitude of the gradient of $\phi$. In fact, it becomes very large near the point of discontinuity. On the other hand, the Hilbert solution, which is described by the Stokes system shown above, is subject to the restriction that the order of magnitude of the spatial derivative should not exceed that of the solution itself (see the sentence following (3.6)). Clearly, this cannot be satisfied uniformly in space due to the condition (3.46). Accordingly, it should be understood that the Stokes system describes the Hilbert solution except in the vicinity of the discontinuity point, although it is solved in the entire domain. It should also be noted that this type of singular condition is often used in conjunction with fluid equations in fluid mechanics. Indeed, the condition (3.46) has the same singularity as the (linearized) Jeffery-Hamel flow with a pair of source and sink in the case where the opening angle between two intersecting planes is $\pi$ (thus, forming a plane). In this sense, the present analysis also sheds light on the interpretation of the diverging singularity frequently encountered in fluid mechanics.

\section{Numerical analysis of a flow driven by a discontinuous surface temperature}

In this section, we carry out a numerical analysis to confirm the results of the present analysis, in particular the macroscopic behavior near the points of discontinuity. The problem we solve is the following.

Let $L$ and $T_{0}$ be the characteristic length and temperature, respectively. Consider a rarefied gas confined in a two-dimensional square vessel with width $L$ (see Fig. 3 ). Let us denote by $L x_{i}$ the space rectangular coordinate system. Then, the walls of the vessel, which are supposed to be at rest, are given by the segments $\left\{x_{1}= \pm 1 / 2,-1 / 2 \leqslant\right.$ $\left.x_{2} \leqslant 1 / 2\right\}$ and $\left\{-1 / 2 \leqslant x_{1} \leqslant 1 / 2, x_{2}= \pm 1 / 2\right\}$. The right-half part $x_{1}>0$ of the vessel is kept at a uniform temperature $T_{0}\left(1+\delta_{\mathrm{w}} / 2\right)$, while the left-half part 
On the motion of slightly rarefied gas induced by a discontinuous surface temperature1

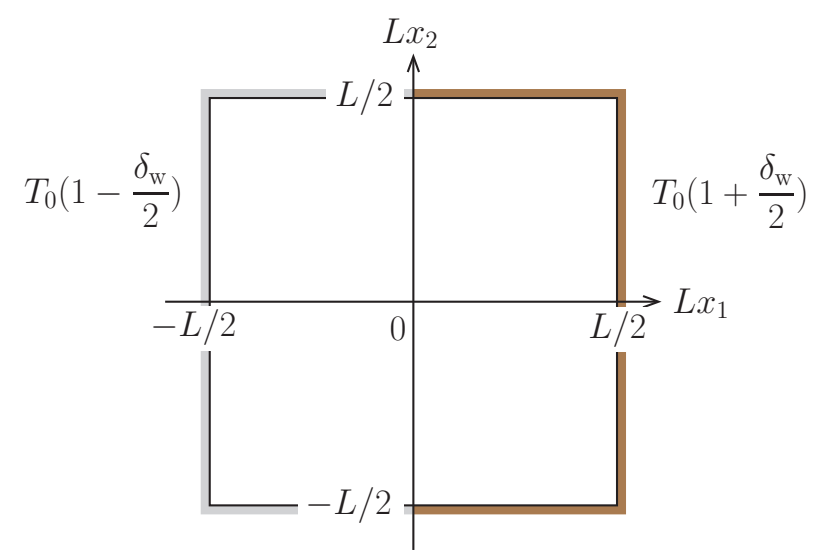

FIgURE 3. A two-dimensional square vessel with a discontinuous surface temperature distribution.

$x_{1}<0$ is kept at another uniform temperature $T_{0}\left(1-\delta_{\mathrm{w}} / 2\right)$, where $\delta_{\mathrm{w}}$ is a positive constant. Thus, the surface temperature distribution along the wall is discontinuous at $\left(x_{1}, x_{2}\right)=(0, \pm 1 / 2)$ with jump $T_{0} \delta_{\mathrm{w}}$. Let $\rho_{0}$ and $p_{0}=\rho_{0} R T_{0}$ be the reference density and pressure, respectively. Investigate the steady behavior of the gas caused by the discontinuous surface temperature based on the Bhatnagar-Gross-Krook (BGK) model of the Boltzmann equation and the diffuse reflection boundary condition, under the assumption that $\delta_{\mathrm{w}}$ is so small that the equations and boundary conditions can be linearized around the reference equilibrium state at rest with temperature $T_{0}$ and density $\rho_{0}$ (or pressure $\left.p_{0}\right)$.

For the BGK model, the mean free path of the gas molecules in the reference equilibrium state at rest is given by $\ell_{0}=(2 / \sqrt{\pi})\left(2 R T_{0}\right)^{1 / 2} /\left(A_{c} \rho_{0}\right)$ with $A_{c}$ being a positive constant such that $A_{c} \rho_{0}$ is the collision frequency in the reference state. Accordingly, the Knudsen number is defined by $K n=\ell_{0} / L$, and as in the foregoing analysis, we mainly use parameter $\varepsilon=(\sqrt{\pi} / 2) K n$ to specify the degree of gas rarefaction. Let us denote by $\left(2 R T_{0}\right)^{1 / 2} \zeta_{i}$ the molecular velocity, by $\rho_{0}\left(2 R T_{0}\right)^{-3 / 2}\left(1+\phi\left(x_{1}, x_{2}, \zeta_{i}\right)\right) E$ the velocity distribution function, by $\rho_{0}\left(1+\omega\left(x_{1}, x_{2}\right)\right)$ the density, by $\left(2 R T_{0}\right)^{1 / 2} u_{i}\left(x_{1}, x_{2}\right)$ the flow velocity $\left(u_{3}=0\right)$, and by $T_{0}\left(1+\tau\left(x_{1}, x_{2}\right)\right)$ the temperature. Then, the linearized BGK equation in the present steady two-dimensional problem is written as

$$
\begin{aligned}
& \zeta_{1} \partial_{1} \phi+\zeta_{2} \partial_{2} \phi=\frac{1}{\varepsilon}\left(-\phi+\omega+2 \zeta_{1} u_{1}+2 \zeta_{2} u_{2}+\left(\zeta_{j}^{2}-\frac{3}{2}\right) \tau\right), \\
& \omega=\langle\phi\rangle, \quad u_{i}=\left\langle\zeta_{i} \phi\right\rangle, \quad(i=1,2), \quad \tau=\frac{2}{3}\left\langle\left(\zeta_{j}^{2}-\frac{3}{2}\right) \phi\right\rangle .
\end{aligned}
$$

The boundary conditions are summarized as

$$
\begin{aligned}
& \phi=-2 \sqrt{\pi} \int_{\zeta_{n}<0} \zeta_{n} \phi E \mathrm{~d} \boldsymbol{\zeta}-\frac{\delta_{\mathrm{w}}}{2}\left(\zeta_{j}^{2}-2\right), \quad \text { for } \quad \zeta_{n}>0, \\
& \left(-\frac{1}{2}<x_{1}<0, x_{2}= \pm \frac{1}{2}\right) \quad \text { or } \quad\left(x_{1}=-\frac{1}{2},-\frac{1}{2}<x_{2}<\frac{1}{2}\right), \\
& \phi=-2 \sqrt{\pi} \int_{\zeta_{n}<0} \zeta_{n} \phi E \mathrm{~d} \zeta+\frac{\delta_{\mathrm{w}}}{2}\left(\zeta_{j}^{2}-2\right), \text { for } \quad \zeta_{n}>0, \\
& \left(0<x_{1}<\frac{1}{2}, x_{2}= \pm \frac{1}{2}\right) \quad \text { or } \quad\left(x_{1}=\frac{1}{2},-\frac{1}{2}<x_{2}<\frac{1}{2}\right),
\end{aligned}
$$

where $\zeta_{n}=\zeta_{i} n_{i}$ and $n_{i}$ is the unit normal vector on the boundary pointed to the gas, 


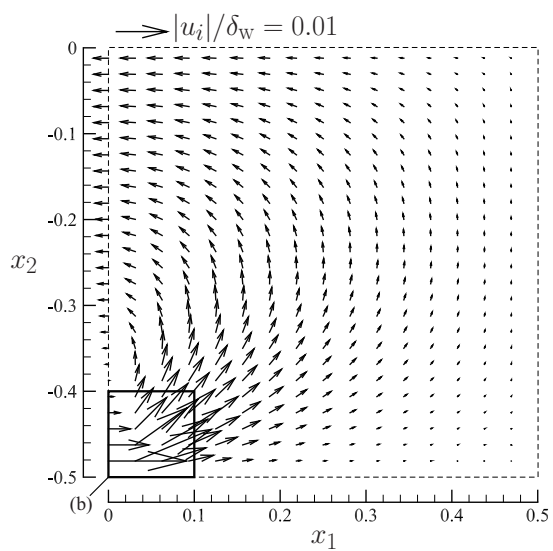

(a)

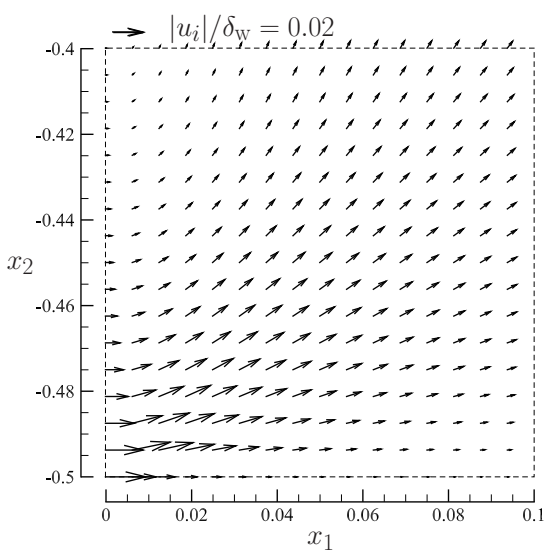

(b)

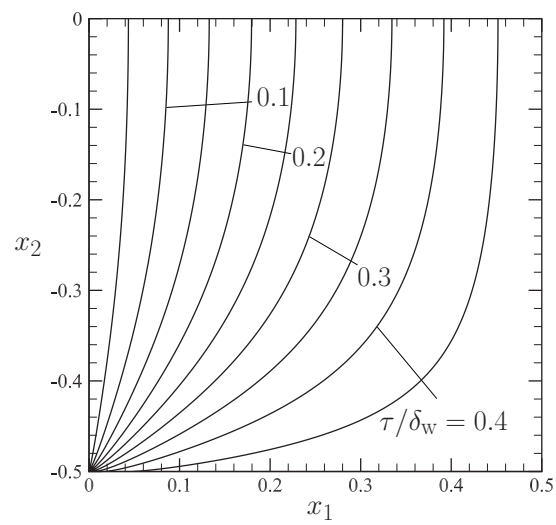

(c)

Figure 4 . The flow velocity $\left(u_{1}, u_{2}\right)$ and temperature $\tau$ in the case of $\varepsilon=0.01$. In panels (a) and (b), the arrow indicates the flow velocity vector $\delta_{\mathrm{w}}^{-1}\left(u_{1}, u_{2}\right)$ at its starting point. The length corresponding to the magnitude $\delta_{\mathrm{w}}^{-1}\left|u_{i}\right|=\delta_{\mathrm{w}}^{-1}\left(u_{1}^{2}+u_{2}^{2}\right)^{1 / 2}=0.01((\mathrm{a}))$ or $0.02((\mathrm{~b}))$ is shown on the top of each panel. Note that the vectors are not shown along $x_{2}=-0.5$ in panel (a). In panel (c), the isothermal lines $\tau / \tau_{\mathrm{w}}=0.05 m(m=0, \ldots, 9)$ are shown.

i.e.,

$$
n_{i}= \begin{cases}(0, \mp 1,0) & \left(-\frac{1}{2} \leqslant x_{1} \leqslant \frac{1}{2}, x_{2}= \pm \frac{1}{2}\right) \\ ( \pm 1,0,0) & \left(x_{1}=\mp \frac{1}{2},-\frac{1}{2}<x_{2}<\frac{1}{2}\right)\end{cases}
$$

Apart from the linearization, the problem is the same as the one investigated in Aoki et al. (2001), where the nonlinear BGK equation was solved numerically (under the diffuse reflection without linearization). Since the linearization is not essential for the numerical treatment, we adopted the same numerical method here. It is a hybrid method using the finite-difference method and the method of characteristics and is designed to capture the propagation of the discontinuity in the phase space. See the reference for further details of the numerical method.

We may assume that the solution $\phi$ is symmetric with respect to the $x_{1}$ axis and 
On the motion of slightly rarefied gas induced by a discontinuous surface temperature3

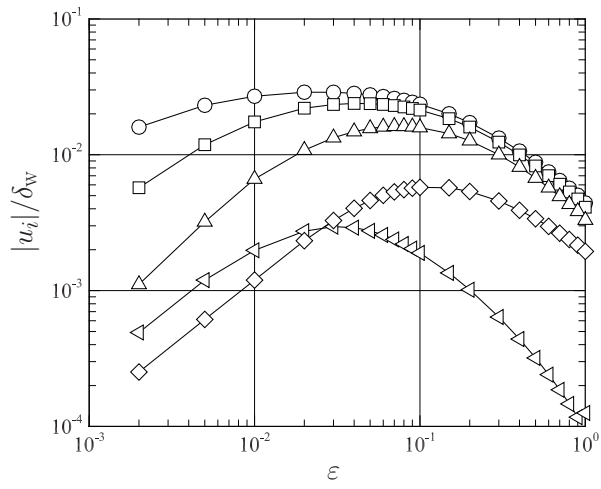

(a)

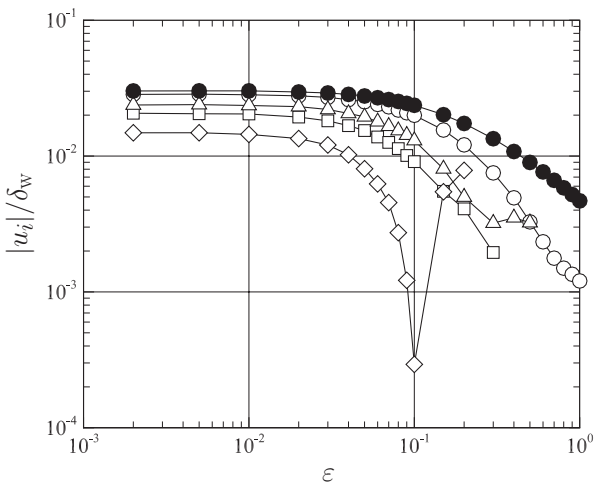

(b)

Figure 5. The flow speed $\left|u_{i}\right|=\left(u_{1}^{2}+u_{2}^{2}\right)^{1 / 2}$ as a function of $\varepsilon$ at various points. (a) $\left|u_{i}\right|$ vs $\varepsilon$ at a fixed point in the domain. $\circ:\left(x_{1}, x_{2}\right)=(0.005,-0.495), \square:(0.02,-0.495), \triangle:(0.05,-0.495)$, $\diamond:(0.3,-0.4), \triangleleft:(0,-0.275)$. (b) $\left|u_{i}\right|$ vs $\varepsilon$ at the points $\left(x_{1}(\varepsilon), x_{2}(\varepsilon)\right)=(a \varepsilon \cos \theta,-1 / 2+a \varepsilon \sin \theta)$. $\circ:(a, \theta)=(1 / 2, \pi / 4), \square:(2, \pi / 4), \triangle:(1, \pi / 3), \diamond:(2, \pi / 2)$. $\bullet$ represents sup $\left|u_{i}\right|$ in the domain.

antisymmetric with respect to the $x_{2}$ axis, namely,

$$
\begin{aligned}
& \phi\left(-x_{1}, x_{2},-\zeta_{1}, \zeta_{2}, \zeta_{3}\right)=\phi\left(x_{1}, x_{2}, \zeta_{1}, \zeta_{2}, \zeta_{3}\right), \\
& \phi\left(x_{1},-x_{2}, \zeta_{1},-\zeta_{2}, \zeta_{3}\right)=-\phi\left(x_{1}, x_{2}, \zeta_{1}, \zeta_{2}, \zeta_{3}\right) .
\end{aligned}
$$

Therefore, the computation was carried out only in the quadrant $0 \leqslant x_{1} \leqslant 1 / 2$ and $-1 / 2 \leqslant x_{2} \leqslant 0$.

For the numerical computation, the domain $\left\{\left(x_{1}, x_{2}\right) \mid 0 \leqslant x_{1} \leqslant 1 / 2,-1 / 2 \leqslant x_{2} \leqslant 0\right\}$ was subdivided into $320 \times 320(160 \times 160)$ nonuniform rectangular meshes for $\varepsilon \leqslant 0.01$ $(\varepsilon>0.01)$. For the molecular velocity, $75 \times 2045\left(\zeta_{r} \times \theta_{\zeta}\right)$ nonuniform grids was used, where $\left(\zeta_{r}, \theta_{\zeta}\right)$ is the polar coorinates for $\left(\zeta_{1}, \zeta_{2}\right)$, i.e., $\zeta_{1}=\zeta_{r} \cos \theta_{\zeta}$ and $\zeta_{2}=\zeta_{r} \sin \theta_{\zeta}$.

In Fig. 4 we show the flow velocity $\left(u_{1}, u_{2}\right)$ and temperature $\tau$ in the case of $\varepsilon=0.01$. A fairly noticeable flow is induced near the point of discontinuity, which drives the overall flow in the container. Near the point of discontinuity, the isolines of the temperature are concentrated, implying that a strong temperature gradient in the clockwise direction is formed near the point. It is interesting to note that these features bear resemblance with the flow around a sharp edge (Taguchi \& Aoki 2012).

To have a closer look at the flow structure, we investigate the magnitude of the flow velocity $\left|u_{i}\right|=\left(u_{1}^{2}+u_{2}^{2}\right)^{1 / 2}$ in two different ways. In the panel (a) of Fig. 5, we plot the variations of the flow speed $\left|u_{i}\right|$ in terms of $\varepsilon$ at five locations in the domain, i.e., $\left(x_{1}, x_{2}\right)=(0.005,-0.495),(0.02,-0.495),(0.05,-0.495),(0.3,-0.4)$, and $(0,-0.275)$. It is seen from the figure that, with the decrease of $\varepsilon$ from 1 to 0.002 , the flow speed increases first, attains the local maximum, and then decreases. Moreover, judging from the slope of the double-log plot, its decay rate for $\epsilon \ll 1$ is likely to be proportional to $\varepsilon$, except in the case $\left(x_{1}, x_{2}\right)=(0.005,-0.495)(\mathrm{o})$. This decaying trend for small $\epsilon$ is consistent with our result $u_{i H}=O(\varepsilon)$ obtained in the analysis of $\S 3$ (see (3.43)). The case $\left(x_{1}, x_{2}\right)=(0.005,-0.495)$ exhibits a slower decay rate, but we expect that the plot will also start to follow the same tendency if $\varepsilon$ is further reduced.

In $\S 3$, we introduced the boundary layer (the Knudsen zone) in the vicinity of the point of discontinuity with the size of the order of $\varepsilon$, where the flow velocity should be $\left|u_{i}\right| / \delta_{\mathrm{w}}=$ $O(1)$. To observe the formation of such a boundary-layer structure in the present problem, 


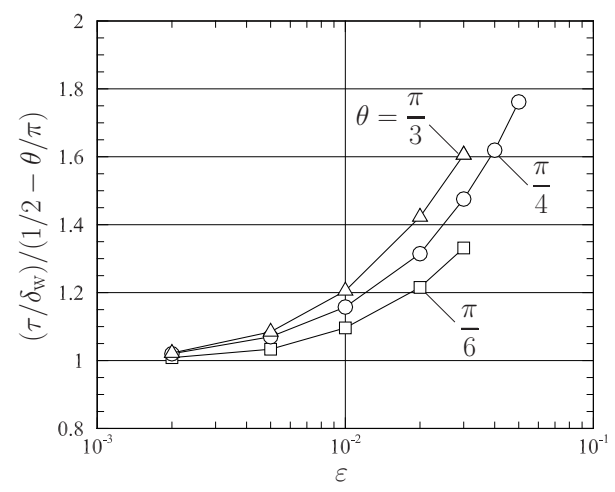

(a)

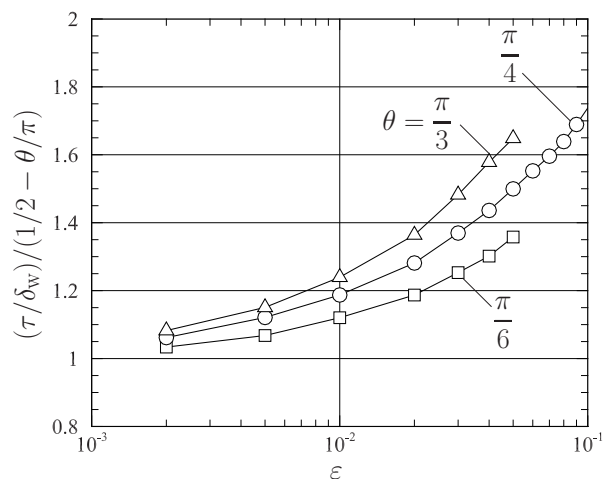

(b)

Figure 6. The normalized temperature $\left(\tau / \delta_{\mathrm{w}}\right) /(1 / 2-\theta / \pi)$ at the point $\left(x_{1}, x_{2}\right)=\left(a \varepsilon^{\alpha} \cos \theta,-1 / 2+a \varepsilon^{\alpha} \sin \theta\right)$ for various $\varepsilon$. (a) $(\alpha, a)=(1 / 2,3)$, (b) $(\alpha, a)=(1 / 3,3 / 2)$. The symbols represent the numerical results, which are connected by the solid lines.

we plot the flow speed $\left|u_{i}\right|$ at the point $\left(x_{1}, x_{2}\right)=(a \varepsilon \cos \theta,-1 / 2+a \varepsilon \sin \theta)$ for various values of $\varepsilon$, for a given set of $a>0$ and $\theta \in[0, \pi / 2]$. The result is shown in the panel (b) of Fig. 5. The figure shows a clear tendency that $\left|u_{i}\right|$ approaches a nonzero limiting value as $\varepsilon$ becomes smaller. Note that the point at which $\left|u_{i}\right|$ is measured approaches the point of discontinuity, but its relative position remains the same in the scale of $\varepsilon$, or in other words, in the scale of the mean free path. This implies that there is a kinetic boundary-layer structure in the vicinity of the discontinuity point, which extends over the distance of the order of $\epsilon$ (or the mean free path).

We mention that essentially the same features were already pointed out in Aoki et al. (2001), where the nonlinear equations were solved. Indeed, Fig. 5 corresponds to Fig. 13 of Aoki et al. (2001). The detailed information provided in that reference served as a basic guideline for the present analysis.

We now turn our attention to the validity of the asymptotic expressions (3.21) and (3.46) for the temperature and the flow velocity. For the present problem, they are expressed as

$$
\frac{\tau}{\delta_{\mathrm{w}}} \sim \frac{1}{2}-\frac{\theta}{\pi}, \quad \frac{u_{r}}{\delta_{\mathrm{w}}} \sim \frac{\varepsilon}{r} \sin 2 \theta, \quad \text { as } r \searrow 0 \text { and } \varepsilon \searrow 0 \text { with } \frac{\varepsilon}{r}=o(1),
$$

where $u_{r}=u_{1} \cos \theta+u_{2} \sin \theta$ is the radial component of the flow velocity around $\left(x_{1}, x_{2}\right)=(0,-1 / 2)$ and $(r, \theta)$ is the local polar coordinate centered at the point of discontinuity, $\left(x_{1}, x_{2}\right)=(r \cos \theta,-1 / 2+r \sin \theta)$. Note that the expression should hold, theoretically, in the region $\varepsilon \ll r \ll 1$.

Let us first consider the temperature $\tau$. We show in Fig. 6 the plot of $\left(\tau / \delta_{\mathrm{w}}\right)(1 / 2-$ $\theta / \pi)^{-1}$ at $\left(x_{1}, x_{2}\right)=\left(a \varepsilon^{\alpha} \cos \theta,-1 / 2+a \varepsilon^{\alpha} \sin \theta\right)$ for various $\varepsilon$ in the cases of $(\alpha, a)=$ $(1 / 2,3)((\mathrm{a}))$ and $(\alpha, a)=(1 / 3,3 / 2)((\mathrm{b}))$. Each panel contains the results for three different values of $\theta(\theta=\pi / 6, \pi / 4, \pi / 3)$ for comparison. Although it is difficult to draw a decisive conclusion, $\left(\tau / \delta_{\mathrm{w}}\right)(1 / 2-\theta / \pi)^{-1}$ is seen to approach the limiting value $(=1)$ with the decrease of $\varepsilon$ irrespective of the values of $\theta$. This supports the asymptotic behavior shown in (4.5).

Finally, we consider the radial component of the flow velocity $u_{r}$. In Fig. 7, we plot $\delta_{\mathrm{w}}^{-1} a\left|u_{r}\right| / \sin 2 \theta$ at $\left(x_{1}, x_{2}\right)=\left(a \varepsilon^{\alpha} \cos \theta,-1 / 2+a \varepsilon^{\alpha} \sin \theta\right)$ for various $\varepsilon$ in the cases of $(\alpha, a)=(1 / 2,1)((\mathrm{a}))$ and $(\alpha, a)=(1 / 3,1 / 2)((\mathrm{b}))$. Again each panel contains the results 
On the motion of slightly rarefied gas induced by a discontinuous surface temperaturi5

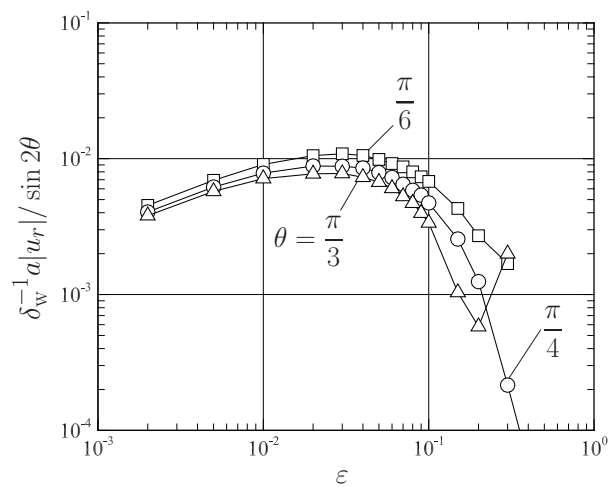

(a)

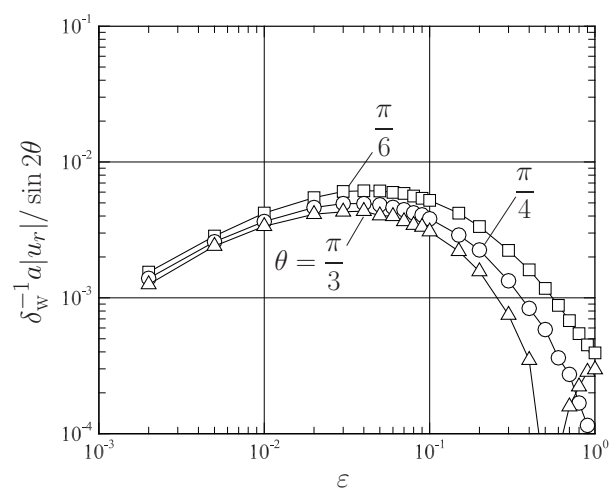

(b)

Figure 7. The normalized radial flow velocity $\delta_{\mathrm{w}}^{-1} a\left|u_{r}\right| / \sin 2 \theta$ at the point $\left(x_{1}(\varepsilon), x_{2}(\varepsilon)\right)=\left(a \varepsilon^{\alpha} \cos \theta,-1 / 2+a \varepsilon^{\alpha} \sin \theta\right)$ for various $\varepsilon$. (a) $(\alpha, a)=(1 / 2,1)$, (b) $(\alpha, a)=(1 / 3,1 / 2)$. See the caption of Fig. 6 .

for three different values of $\theta(\theta=\pi / 6, \pi / 4, \pi / 3)$. If the asymptotic behavior shown in (4.5) holds, the double-log plot of $\delta_{\mathrm{w}}^{-1} a\left|u_{r}\right| / \sin 2 \theta$ as a function of $\varepsilon$ for a given $(\alpha, a, \theta)$ should approach a line with the slope $1-\alpha$ as $\varepsilon \searrow 0$. In Fig. 7, we actually observe that each plot tends to approach a line with the slope $1 / 2$ in (a) and a line with the slope $2 / 3$ in (b), independently of $\theta$, as $\varepsilon$ is reduced. This supports again the asymptotic behavior of $u_{r}$ shown in (4.5).

To summarize, the numerical results given above support the presence of the crossover region between the Knudsen zone and the outer region in which the macroscopic variables behave like $\tau / \delta_{\mathrm{w}} \sim(1 / 2-\theta / \pi)$ and $u_{r} / \delta_{\mathrm{w}} \sim(\varepsilon / r) \sin 2 \theta$.

\section{Concluding remarks}

In this paper, we considered a slightly rarefied gas confined between two parallel plates with a discontinuous surface temperature distribution and investigated its steady behavior. We aimed at extending the conventional GSF theory, which assumes smooth boundary data, to the case in which the boundary temperature has a jump discontinuity, in the simplest case where the boundary is a flat surface. With the aid of rich 
accumulation of the results known for the case of smooth boundary data, we carried out an asymptotic analysis of the Boltzmann system for small Knudsen numbers based on the matched asymptotic expansion, which employs Sone's asymptotics as the outer solution and a newly introduced kinetic boundary later (the Knudsen zone) as the inner solution. The analysis was carried out to the first order of the Knudsen number and in particular, an appropriate form of the "slip boundary condition" for the flow driven by a discontinuous surface temperature was identified. The main results obtained in this paper are summarized as follows:

- The conventional slip flow theory which assumes the smoothness of the boundary data breaks down near the point where the surface temperature is discontinuous. In this local region (or the Knudsen zone), the solution varies in the scale of the mean free path in both normal and tangential directions to the boundary.

- The half-space boundary-value problem associated with the Knudsen zone for a discontinuous surface temperature over a flat boundary was formulated. The asymptotic form of the solution at far field was derived.

- In the Knudsen zone, the flow velocity decreases with distance in proportion to the reciprocal of the distance. In the far-field of the Knudsen zone, the flow velocity has radially diverging or converging streamlines depending on the hotter or colder side.

- The overall flow velocity in the channel is expressed as $u_{i}=\varepsilon u_{i H 1}+O\left(\varepsilon^{2}\right)$ and the essential term $u_{i H 1}$ is described by the Stokes equation for an incompressible fluid with the no-slip boundary condition. It also satisfies a singular source/sink condition located at the point of discontinuity, which diverges in approaching the point of discontinuity. The analysis of the half-space problem (i.e., the Knudsen-zone problem) mentioned above determines the multiplicative factor $\left(\Gamma_{Z}^{(1)}\right)$ of this singular source/sink condition.

- The result of the direct numerical analysis based on the BGK model for a rarefied gas flow in a square vessel with a discontinuous surface temperature is consistent with that of the asymptotic analysis.

In this way, the "slip boundary condition" for the flow induced by a discontinuous surface temperature is qualitatively different from those for the thermal creep or thermal stress slip flow.

This work was supported by JSPS KAKENHI grant No. 17K06146. The authors thank Mr. Kanji Oniyama for his assistance in the numerical analysis carried out in $\S 4$. A part of numerical computations was carried out using the supercomputer of ACCMS, Kyoto University.

Declaration of Interests. The authors report no conflict of interest.

\section{Appendix A. Derivation of (3.34) and (3.35)}

In this appendix, we outline the derivation of (3.34) and (3.35). We focus on the derivation of the Hilbert part, since the derivation of the Knudsen-layer part is parallel to the derivation of boundary conditions for the Stokes system. Throughout this appendix, the subscript $Z 0$ will be omitted.

Let us assume that, for some small constant $0<\delta \ll 1$, the length scale of variation is $\delta^{-1}$ in the region $\left(y_{1}^{2}+y_{2}^{2}\right)^{1 / 2}>\delta^{-1}$, i.e., $\partial \phi / \partial y_{i}=O(\delta \phi)$. The validity of this assumption is verified if such a solution is obtained. Then, introducing new variables

$$
x_{i}=\delta y_{i},
$$

the velocity distribution function is sought in the form $\phi=\phi\left(x_{1}, x_{2}, \xi_{i}\right)$. Then, $\phi$ satisfies 
On the motion of slightly rarefied gas induced by a discontinuous surface temperature7 the linearized Boltzmann equation of the form

$$
\xi_{1} \partial_{1} \phi+\xi_{2} \partial_{2} \phi=\frac{1}{\delta} \mathcal{L}(\phi)
$$

Thus, $\delta$ plays the role of a small Knudsen number and one can seek an asymptotic solution for small values of $\delta$ in the form

$$
\begin{aligned}
& \phi=\phi_{H}+\phi_{K}, \\
& \phi_{H}=\phi_{H 0}+\phi_{H 1} \delta+\cdots, \\
& \phi_{K}=\phi_{K 1} \delta+\cdots,
\end{aligned}
$$

where $\phi_{H}$ is the Hilbert solution and $\phi_{K}$ is the Knudsen-layer correction near the boundary $x_{1}=0$. The corresponding macroscopic quantities $\omega, u_{i}, \tau$, and $P$ are expressed similarly as

$$
\begin{aligned}
& h=h_{H}+h_{K}, \\
& h_{H}=h_{H 0}+h_{H 1} \delta+\cdots, \\
& h_{K}=h_{K 1} \delta+\cdots,
\end{aligned}
$$

where $h=\omega, u_{i}, \tau$, and $P$. Then, the analysis is analogous to $\S 2(\delta$ corresponds to $\varepsilon)$. In particular, the boundary conditions for the flow velocity and temperature on the lower half of the boundary, $x_{1}=0$ and $x_{2}<0$, are obtained from those on the upper half by replacing $\tau_{\mathrm{w}}$ by $-\tau_{\mathrm{w}}$. We shall obtain the asymptotic solution satisfying the required symmetry (3.32).

\section{Order $\delta^{0}$}

We solve equations $(2.11)-(2.12)$ for $m=0$ with $\partial_{3}=0$ and $u_{3 H 0}=0$ under the boundary conditions

$$
\begin{aligned}
& u_{i H 0}=0, \quad \tau_{H 0}= \pm \tau_{\mathrm{w}}, \quad\left(x_{1}=0, \pm x_{2}>0\right), \\
& u_{i H 0} \rightarrow 0, \quad \tau_{H 0} \rightarrow \frac{2 \tau_{\mathrm{w}}}{\pi} \theta, \quad P_{H 0} \rightarrow 0, \quad(r \rightarrow \infty),
\end{aligned}
$$

where $r=\sqrt{x_{1}^{2}+x_{2}^{2}}$ and $\theta=\operatorname{Arctan}\left(x_{2} / x_{1}\right)$. The solution is given by

$$
P_{H 0}=0, \quad u_{i H 0}=0, \quad P_{H 1}=0, \quad \tau_{H 0}=\frac{2 \tau_{\mathrm{w}}}{\pi} \theta,
$$

where $P_{H 1}$ is determined up to an additive constant. Thus, the velocity distribution function is obtained as

$$
\phi_{H 0}=\frac{2 \tau_{\mathrm{w}}}{\pi}\left(\xi_{j}^{2}-\frac{5}{2}\right) \theta
$$

There is no Knudsen-layer correction required at this order.

Order $\delta^{1}$

We solve equations (2.12) for $m=1$ with $\partial_{3}=0$ and $u_{3 H 1}=0$ under the boundary conditions

$$
\begin{aligned}
& u_{r H 1}=u_{\theta H 1}=0, \quad \tau_{H 1}=\mp \frac{2 \tau_{\mathrm{w}} c_{1}^{(0)}}{\pi} \frac{1}{r}, \quad\left(\theta= \pm \frac{\pi}{2}, r>0\right), \\
& u_{i H 1} \rightarrow 0, \quad \tau_{H 1} \rightarrow 0, \quad P_{H 1} \rightarrow 0, \quad(r \rightarrow \infty),
\end{aligned}
$$


where $u_{r H m}$ and $u_{\theta H m}$ are the radial and circumferential components of $u_{i H m}$ in the polar coordinate system centered at the origin. From the last condition of $(\mathrm{A} 8 b)$, the additive constant in $P_{H 1}$ must vanish. Solving the problem, we have

$$
\begin{aligned}
& P_{H 1}=0, \quad u_{r H 1}=\tau_{\mathrm{w}} \frac{\Gamma_{Z}^{(1)} \sin 2 \theta}{r}, \quad u_{\theta H 1}=0, \\
& P_{H 2}=\tau_{\mathrm{w}} \frac{2 \gamma_{1} \Gamma_{Z}^{(1)}}{r^{2}} \sin 2 \theta, \quad \tau_{H 1}=-\frac{2}{\pi} \tau_{\mathrm{w}} c_{1}^{(0)} \frac{\sin \theta}{r},
\end{aligned}
$$

where $\Gamma_{Z}^{(1)}$ is an undetermined constant and $P_{H 2}$ is determined up to an additive constant. From (2.43), the velocity distribution function is given by

$$
\begin{aligned}
\frac{\phi_{H 1}}{\tau_{\mathrm{w}}} & =2 \xi_{r} \frac{\Gamma_{Z}^{(1)} \sin 2 \theta}{r}-\left(\xi_{j}^{2}-\frac{5}{2}\right) \frac{2}{\pi} c_{1}^{(0)} \frac{\sin \theta}{r}-\frac{2}{\pi} A(\xi) \frac{\xi_{\theta}}{r}, \\
\frac{\phi_{K 1}}{\tau_{\mathrm{w}}} & =-\frac{2}{\pi} \frac{1}{x_{2}} \varphi_{1}^{(0)}\left(y_{1}, \xi_{1}, \xi\right),
\end{aligned}
$$

where $\xi=\left(\xi_{j}^{2}\right)^{1 / 2}$ and $y_{1}=x_{1} / \delta$. The Knudsen-layer corrections for the macroscopic quantities are summarized as

$$
\begin{aligned}
& u_{r K 1}=u_{\theta K 1}=0, \quad \omega_{K 1}=-\frac{2}{\pi} \frac{1}{x_{2}} \Omega_{1}^{(0)}\left(y_{1}\right), \\
& \tau_{K 1}=-\frac{2}{\pi} \frac{1}{x_{2}} \Theta_{1}^{(0)}\left(y_{1}\right), \quad P_{K 1}=-\frac{2}{\pi} \frac{1}{x_{2}}\left(\Omega_{1}^{(0)}\left(y_{1}\right)+\Theta_{1}^{(0)}\left(y_{1}\right)\right) .
\end{aligned}
$$

Order $\delta^{2}$

For the present purpose of the study, we do not need the information of the far-field asymptotics to the order $\delta^{2}$. However, it is important for the numerical analysis as well as for a theoretical insight. Therefore, we present the result.

If we continue the analysis in $\S 2$ to the order of $\varepsilon^{2}$ or referring to Chap. 3 of Sone (2007) (or to Takata \& Hattori (2012)), the boundary conditions on $x_{1}=0$ are given by

$$
\begin{aligned}
& u_{r H 2}= \pm 2 \tau_{\mathrm{w}} \frac{\Gamma_{Z}^{(1)} b_{1}^{(1)}+\frac{c_{1}^{(0)} b_{2}^{(1)}+b_{3}^{(1)}}{\pi}}{r^{2}}, \quad u_{\theta H 2}=0, \quad \tau_{H 2}=0, \quad\left(\theta= \pm \frac{\pi}{2}, r>0\right), \\
& u_{i H 2} \rightarrow 0, \quad \tau_{H 2} \rightarrow 0, \quad P_{H 2} \rightarrow 0, \quad(r \rightarrow \infty) .
\end{aligned}
$$

The last condition of (A $12 b$ ) determines the additive constant in $P_{H 2}$ to be zero. Finding solutions to (2.12) for $m=2$ with $\partial_{3}=0$ and $u_{3 H_{2}}=0$ under these conditions, we have

$$
\begin{aligned}
& \frac{u_{r H 2}}{\tau_{\mathrm{w}}}=\frac{-3 \Gamma_{Z}^{(2)}(\sin 3 \theta+\sin \theta)+2\left(\Gamma_{Z}^{(1)} b_{1}^{(1)}+\frac{c_{1}^{(0)} b_{2}^{(1)}+b_{3}^{(1)}}{\pi}\right) \sin \theta}{r^{2}}, \\
& \frac{u_{\theta H 2}}{\tau_{\mathrm{w}}}=\frac{\Gamma_{Z}^{(2)}(\cos 3 \theta+3 \cos \theta)-2\left(\Gamma_{Z}^{(1)} b_{1}^{(1)}+\frac{c_{1}^{(0)} b_{2}^{(1)}+b_{3}^{(1)}}{\pi}\right) \cos \theta}{r^{2}}, \\
& \frac{\tau_{H 2}}{\tau_{\mathrm{w}}}=\frac{\Lambda_{Z}^{(2)}}{r^{2}} \sin 2 \theta,
\end{aligned}
$$

where $\Gamma_{Z}^{(2)}$ and $\Lambda_{Z}^{(2)}$ are undetermined constants. It is worth noting that the flow velocity is a special case of plane flows around a corner (Moffatt 1964). The velocity distribution 
On the motion of slightly rarefied gas induced by a discontinuous surface temperature9 function is then given by

$$
\begin{aligned}
\frac{\phi_{H 2}}{\tau_{\mathrm{w}}} & =\frac{2 \gamma_{1} \Gamma_{Z}^{(1)}}{r^{2}} \sin 2 \theta+\left(\xi_{j}^{2}-\frac{5}{2}\right) \frac{\Lambda_{Z}^{(2)}}{r^{2}} \sin 2 \theta \\
& -2 \Gamma_{Z}^{(2)} \frac{3 \xi_{r}(\sin 3 \theta+\sin \theta)-\xi_{\theta}(\cos 3 \theta+3 \cos \theta)}{r^{2}} \\
& +4\left(\Gamma_{Z}^{(1)} b_{1}^{(1)}+\frac{c_{1}^{(0)} b_{2}^{(1)}+b_{3}^{(1)}}{\pi}\right) \frac{\xi_{r} \sin \theta-\xi_{\theta} \cos \theta}{r^{2}} \\
& -\frac{2}{\pi} c_{1}^{(0)}\left(\frac{\xi_{r} \sin \theta-\xi_{\theta} \cos \theta}{r^{2}}\right) A(\xi) \\
& +\Gamma_{Z}^{(1)}\left(\frac{\left(\xi_{r}^{2}-\xi_{\theta}^{2}\right) \sin 2 \theta-2 \xi_{r} \xi_{\theta} \cos 2 \theta}{r^{2}}\right) B(\xi)+\frac{4}{\pi} \frac{\xi_{r} \xi_{\theta}}{r^{2}} F(\xi), \\
\frac{\phi_{K 2}}{\tau_{\mathrm{w}}} & =\frac{\xi_{2}}{x_{2}^{2}}\left(2 \Gamma_{Z}^{(1)} \varphi_{1}^{(1)}\left(y_{1}, \xi_{1}, \xi\right)+\frac{2}{\pi} c_{1}^{(0)} \varphi_{2}^{(1)}\left(y_{1}, \xi_{1}, \xi\right)+\frac{2}{\pi} \varphi_{3}^{(1)}\left(y_{1}, \xi_{1}, \xi\right)\right) .
\end{aligned}
$$

The Knudsen-layer corrections for the macroscopic quantities are summarized as

$$
\begin{aligned}
& \frac{u_{r K 2}}{\tau_{\mathrm{w}}}=\frac{2}{x_{2}^{2}}\left(\Gamma_{Z}^{(1)} Y_{1}^{(1)}\left(y_{1}\right)+\frac{c_{1}^{(0)} Y_{2}^{(1)}\left(y_{1}\right)+Y_{3}^{(1)}\left(y_{1}\right)}{\pi}\right) \sin \theta, \\
& \frac{u_{\theta K 2}}{\tau_{\mathrm{w}}}=\frac{2}{x_{2}^{2}}\left(\Gamma_{Z}^{(1)} Y_{1}^{(1)}\left(y_{1}\right)+\frac{c_{1}^{(0)} Y_{2}^{(1)}\left(y_{1}\right)+Y_{3}^{(1)}\left(y_{1}\right)}{\pi}\right) \cos \theta, \\
& \omega_{K 2}=\tau_{K 2}=P_{K 2}=0 .
\end{aligned}
$$

Assembling the above results according to (A 3)-(A 4) and taking account the relations $\delta / x_{2}=1 / y_{2}$ and $\delta / r=1 / \widetilde{r}$, we obtain (3.34) and (3.35).

\section{REFERENCES}

Andries, P., Tallec, P. Le, Perlat, J.-P. \& Perthame, B. 2000 The Gaussian-BGK model of Boltzmann equation with small Prandtl number. Eur. J. Mech. B/Fluids 19 (6), 813830.

Aoki, K., Sone, Y. \& Masukawa, N. 1995 A rarefied gas flow induced by a temperature field. In Rarefied Gas Dynamics (ed. J. Harvey \& G. Lord), pp. 35-41. Oxford: Oxford Univ. Press.

Aoki, K., Takata, S., Aikawa, H. \& Golse, F. 2001 A rarefied gas flow caused by a discontinuous wall temperature. Phys. Fluids 13 (9), 2645-2661, erratum: ibid. 13, 3843 (2001).

Aoki, K., Tаката, S. \& Tомота, T. 2014 A force acting on an oblate spheroid with discontinuous surface temperature in a slightly rarefied gas. J. Fluid Mech. 748, 712730.

Baier, T., Hardt, S., Shahabi, V. \& Roohi, E. 2017 Knudsen pump inspired by Crookes radiometer with a specular wall. Phys. Rev. Fluids 2, 033401.

Baier, T., Tiwari, S., Shrestha, S., Klar, A. \& Hardt, S. 2018 Thermophoresis of Janus particles at large Knudsen numbers. Phys. Rev. Fluids 3, 094202.

Bardos, C., Caflisch, R. E. \& Nicolaenko, B. 1986 The Milne and Kramers problems for the Boltzmann equation of a hard sphere gas. Commun. Pure Appl. Math. 39 (3), 323-352.

Bhatnagar, P. L., Gross, E. P. \& Krook, M. 1954 A model for collision processes in gases. I. Small amplitude processes in charged and neutral one-component systems. Phys. Rev. 94, 511-525. 
Brull, S. 2015 An ellipsoidal statistical model for gas mixtures. Commun. Math. Sci. 13 (1), $1-13$.

Coron, F., Golse, F. \& Sulem, C. 1988 A classification of well-posed kinetic layer problems. Commun. Pure Appl. Math. 41 (4), 409-435.

Donkov, A. A., Tiwari, S., Liang, T., Hardt, S., Klar, A. \& Ye, W. 2011 Momentum and mass fluxes in a gas confined between periodically structured surfaces at different temperatures. Phys. Rev. E 84, 016304.

Dyke, M. Van 1975 Perturbation Methods in Fluid Mechanics. Stanford: Parabolic Press.

HolwaY, J. 1966 New statistical models for kinetic theory: Methods of construction. Phys. Fluids 9 (9), 1658-1673.

Kennard, E. H. 1938 Kinetic Theory of Gases. New York: McGraw-Hill.

Ketsdever, A., Gimelshein, N., Gimelshein, S. \& Selden, N. 2012 Radiometric phenomena: From the 19th to the 21st century. Vacuum 86, 1644-1662.

Li, Q., Liang, T. \& Ye, W. 2014 Knudsen torque: A rotational mechanism driven by thermal force. Phys. Rev. E 90, 033009.

Lotfian, A. \& Roohi, E. 2019 Radiometric flow in periodically patterned channels: fluid physics and improved configurations. J. Fluid Mech. 860, 544-576.

Loyalka, S. K. 1969 Thermal transpiration in a cylindrical tube. Phys. Fluids 12, 2301-2305.

Moffatt, H. K. 1964 Viscous and resistive eddies near a sharp corner. J. Fluid Mech. 18 (1), $1-18$.

Niımi, H. 1971 Thermal creep flow of rarefied gas between two parallel plates. J. Phys. Soc. Jpn. 30 (2), 572-574.

Ohwada, T., Sone, Y. \& Aoki, K. 1989a Numerical analysis of the Poiseuille and thermal transpiration flows between two parallel plates on the basis of the Boltzmann equation for hard-sphere molecules. Phys. Fluids A 1 (12), 2042-2049, erratum: ibid. 2, 639 (1990).

Ohwada, T., Sone, Y. \& Aoki, K. $1989 b$ Numerical analysis of the shear and thermal creep flows of a rarefied gas over a plane wall on the basis of the linearized Boltzmann equation for hard-sphere molecules. Phys. Fluids A 1 (9), 1588-1599.

Sharipov, F. 2002 Application of the Cercignani-Lampis scattering kernel to calculations of rarefied gas flows. III. Poiseuille flow and thermal creep through a long tube. Eur. J. Mech. B/Fluids 22 (2), 145-154.

Sone, Y. 1966 Thermal creep in rarefied gas. J. Phys. Soc. Jpn. 21, 1836-1837.

Sone, Y. 1969 Asymptotic theory of flow of rarefied gas over a smooth boundary I. In Rarefied Gas Dynamics (ed. L. Trilling \& H. Y. Wachman), vol. 1, pp. 243-253. New York: Academic Press.

Sone, Y. 1971 Asymptotic theory of flow of rarefied gas over a smooth boundary II. In Rarefied Gas Dynamics (ed. D. Dini), vol. 2, pp. 737-749. Pisa: Editrice Tecnico Scientfica.

Sone, Y. 2002 Kinetic Theory and Fluid Dynamics. Boston: Birkhäuser, Supplementary Notes and Errata: Kyoto University Research Information Repository (http://hdl.handle.net/2433/66099).

Sone, Y. 2007 Molecular Gas Dynamics: Theory, Techniques, and Applications. Boston: Birkhäuser, Supplementary Notes and Errata: Kyoto University Research Information Repository (http://hdl.handle.net/2433/66098).

Sone, Y. \& Yamamoto, K. 1968 Flow of rarefied gas through a circular pipe. Phys. Fluids 11, 1672-1678, erratum: ibid. 13, 1651 (1970).

Sone, Y. \& Yoshimoto, M. 1997 Demonstration of a rarefied gas flow induced near the edge of a uniformly heated plate. Phys. Fluids 9, 3530-3534, Supplementary Video: Kyoto University Research Information Repository (http://hdl.handle.net/2433/122357).

Sugimoto, H. \& Sone, Y. 2005 Vacuum pump without a moving part by thermal edge flow. In Rarefied Gas Dynamics (ed. M. Capitelli), pp. 168-173. Melville: AIP.

TAGUCHI, S. 2015 Asymptotic theory of a uniform flow of a rarefied gas past a sphere at low Mach numbers. J. Fluid Mech. 774, 363-394.

TAguchi, S. \& Aoki, K. 2012 Rarefied gas flow around a sharp edge induced by a temperature field. J. Fluid Mech. 694, 191-224.

TAGUCHI, S. \& Aoki, K. 2015 Motion of an array of plates in a rarefied gas caused by radiometric force. Phys. Rev. E 91, 063007. 
On the motion of slightly rarefied gas induced by a discontinuous surface temperatur:1

TAguchi, S. \& Suzuki, T. 2017 Asymptotic far-field behavior of macroscopic quantities in a problem of slow uniform rarefied gas flow past a sphere. Phys. Rev. Fluids 2, 113401.

TAkata, S. \& Funagane, H. 2011 Poiseuille and thermal transpiration flows of a highly rarefied gas: Over-concentration in the velocity distribution function. J. Fluid Mech. 669, 242-259.

TAkata, S. \& Hattori, M. 2012 Asymptotic theory for the time-dependent behavior of a slightly rarefied gas over a smooth solid boundary. J. Stat. Phys. 147 (6), 1182-1215.

TAKata, S. \& HATtori, M. 2015 Numerical data for the generalized slip-flow theory. Kyoto University Research Information Repository (http://hdl.handle.net/2433/199811).

Takata, S., Hattori, M. \& Hasebe, T. 2016 Slip/jump coefficients and Knudsen-layer corrections for the ES model in the generalized slip-flow theory. AIP Conf. Proc. 1786 (1), 040004 .

Welander, P. 1954 On the temperature jump in a rarefied gas. Ark. Fys. 7, 507-553. 